فاعلية التدريب المتباين على مستوى بعض القدر ات الحركية الخاصة ومحددات النشاط الهجومى للدى لاعبى مسـابقة القتال الفعلي "الكوميته" برياضة النئ الكار اتيه.

قسم الرياضات المائية والمنازلات، كلية التربية الرياضية للبنات، جامعة الزقازيق - جمهورية مصر العربية.

المقدمة ومشكلة البحث

تتضافر جهود الباحثين فى مجال التدريب الرياضى فى جميع الأنشطة البدنية نحو العمل على تحسين وتطوير الأداء فى نوع على النشـاط الممارس

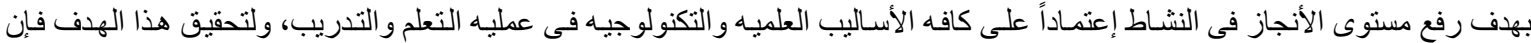

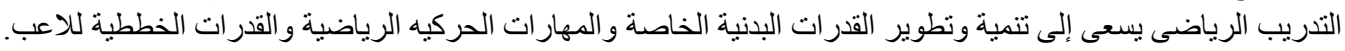

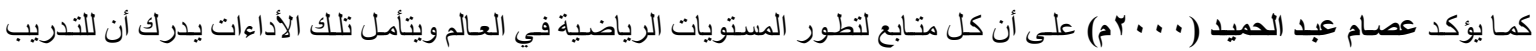

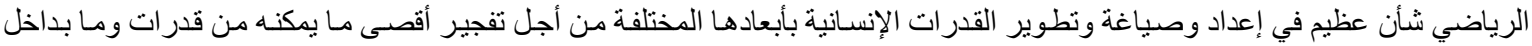

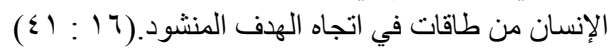

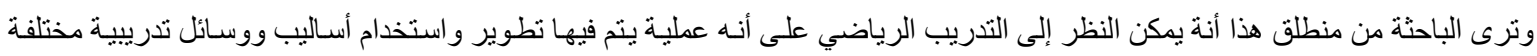

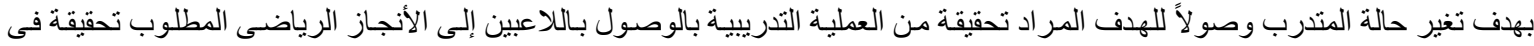
المسابقات الرياضية المختلفة.

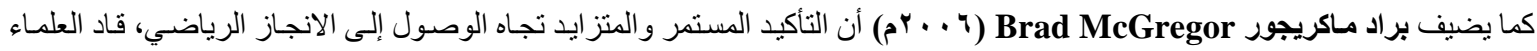

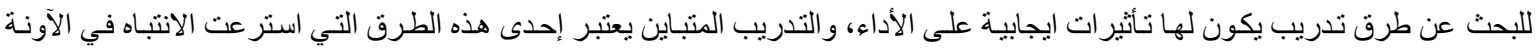

الأخيرة.(1) : (

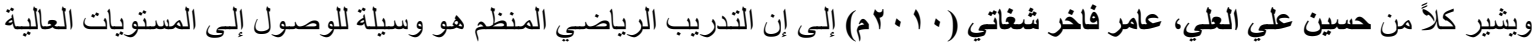

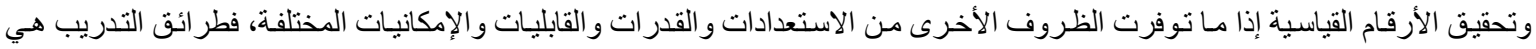

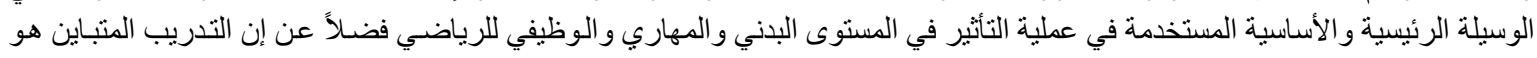

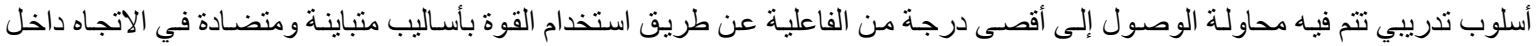

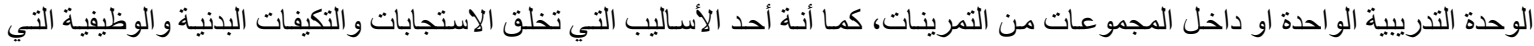

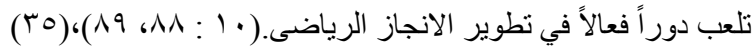

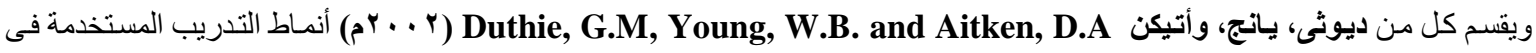

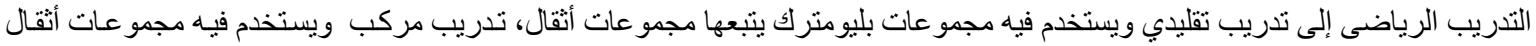

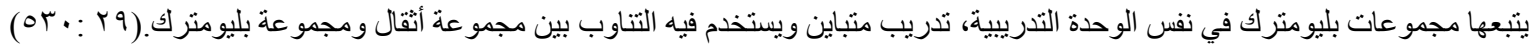

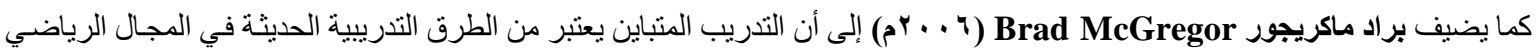

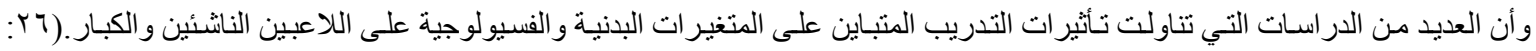

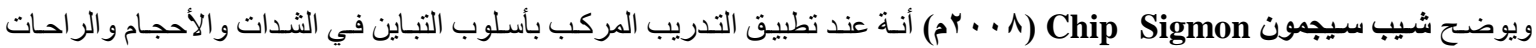

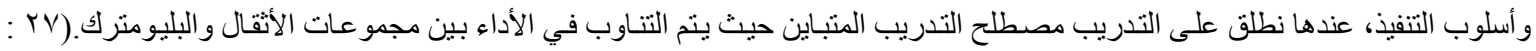

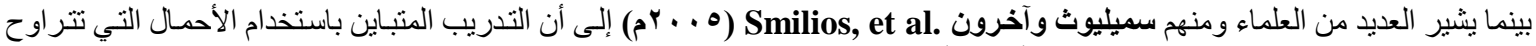

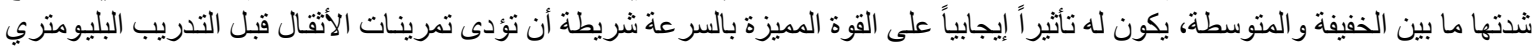

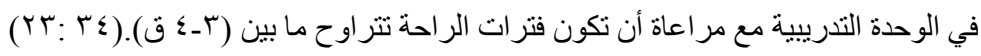

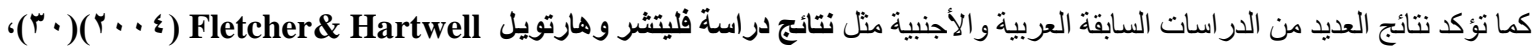

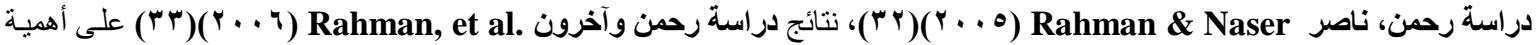

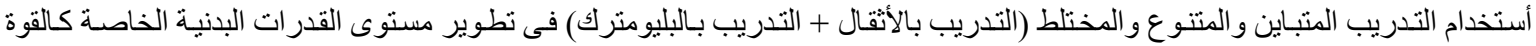

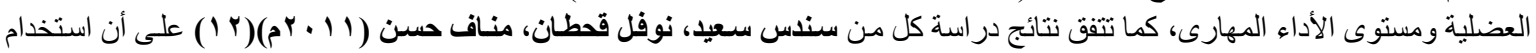


تدريبات الأثقال و البليو مترك بأسلوب متباين يساهم مساهمة فعالة وإيجابية فى تطوير قوة عضلات التبات الرجلين للاعبين، و على ضلى ضرورة التركيز

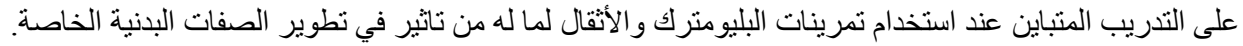

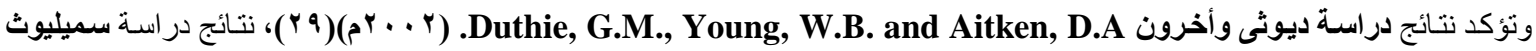

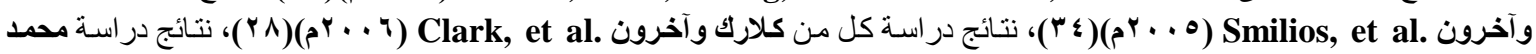

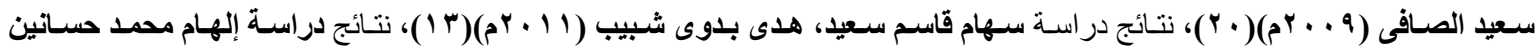

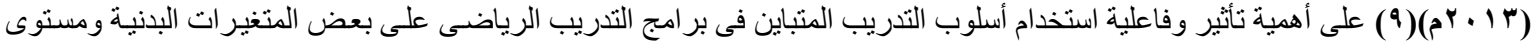

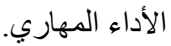

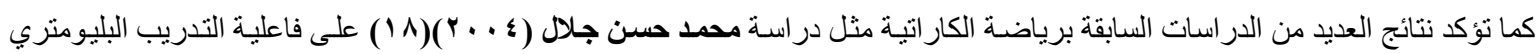

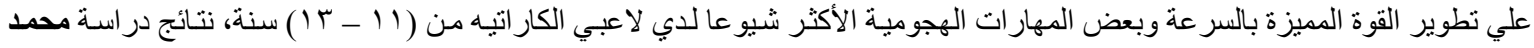

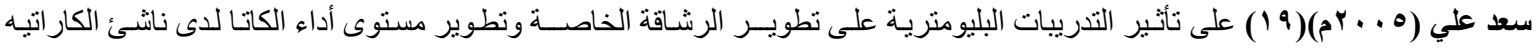

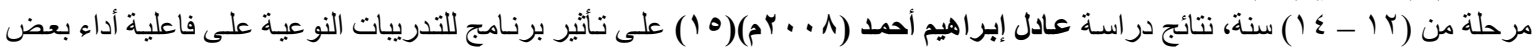

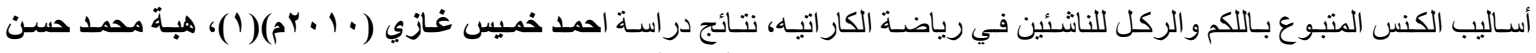

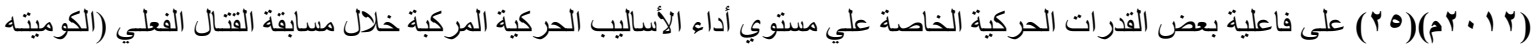

(kumite -

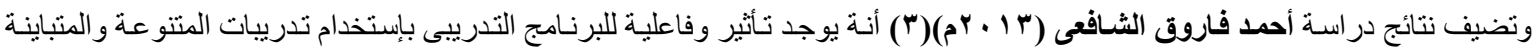

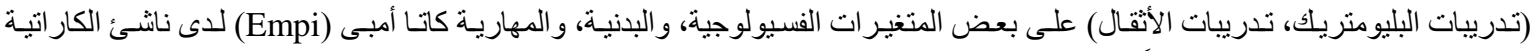

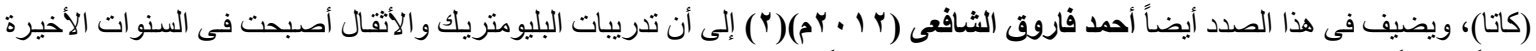

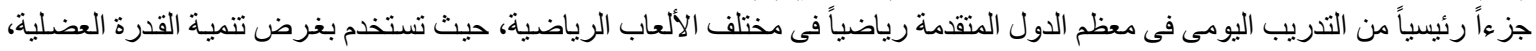

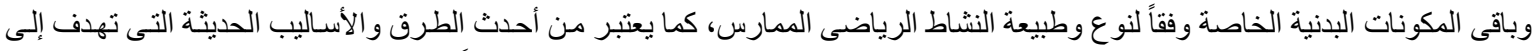

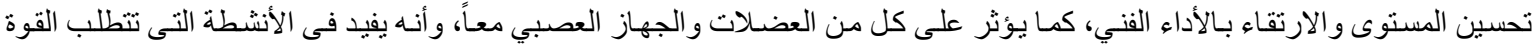

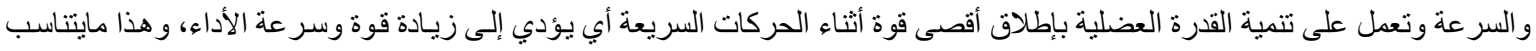

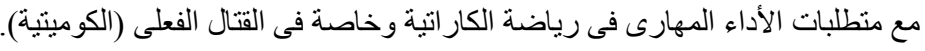

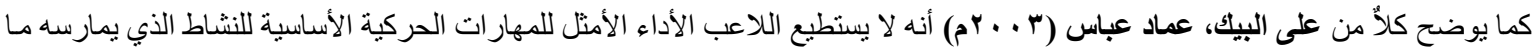

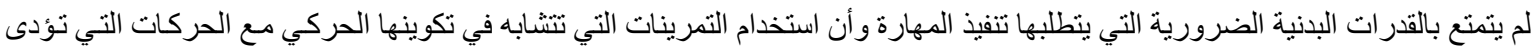

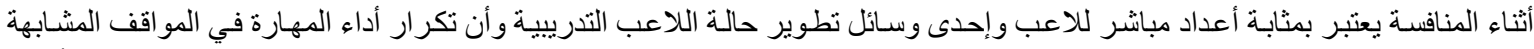

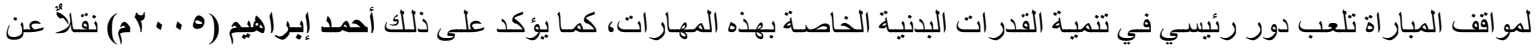

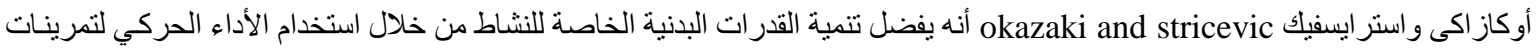

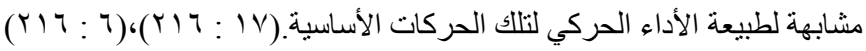

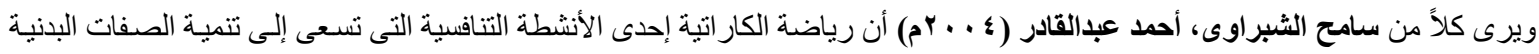

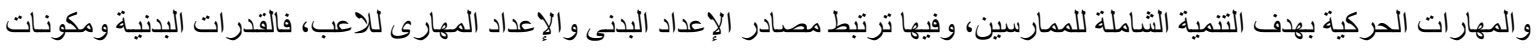

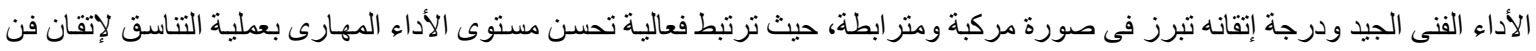

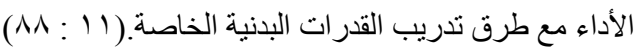

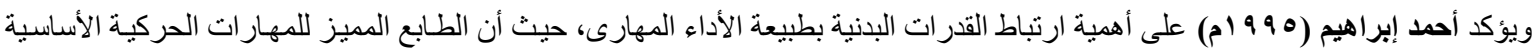

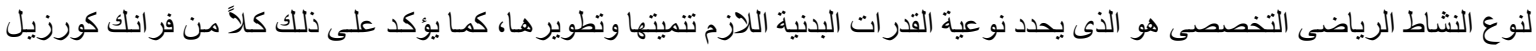

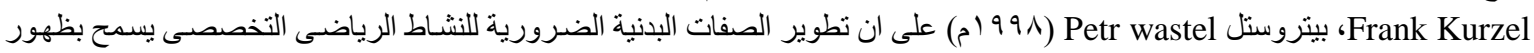

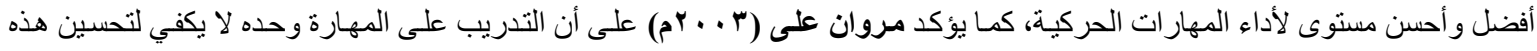

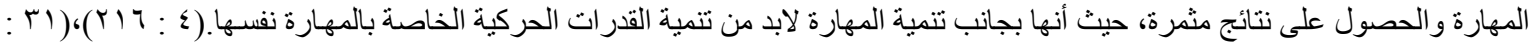

$(r \varepsilon) \cdot(9)$

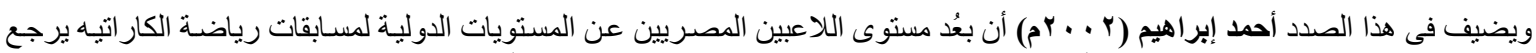

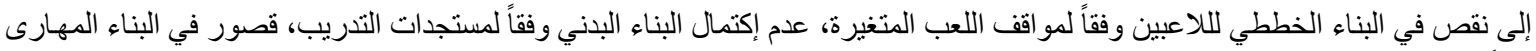

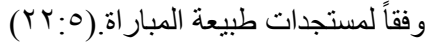

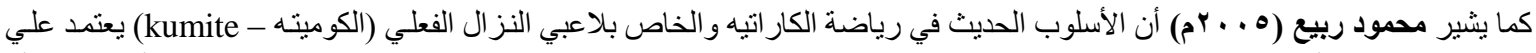

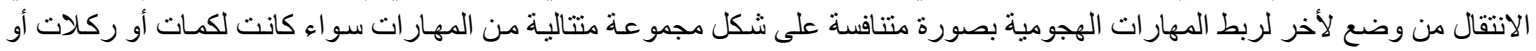

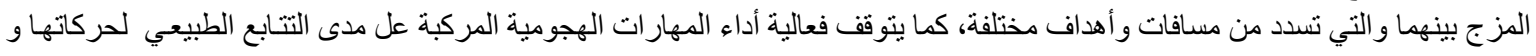




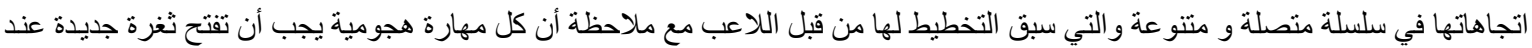

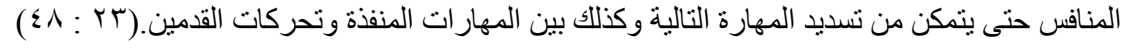

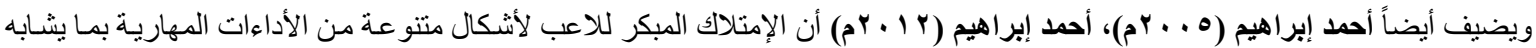

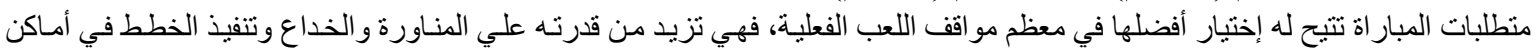

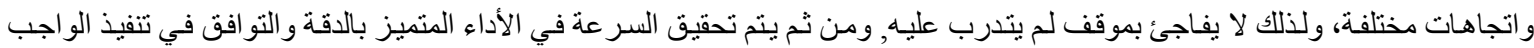

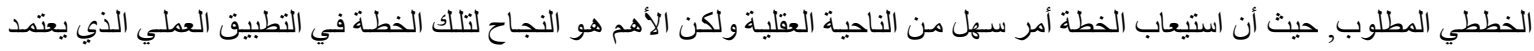

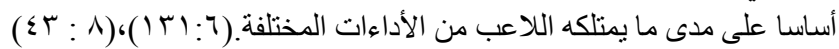

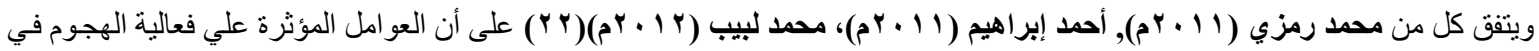

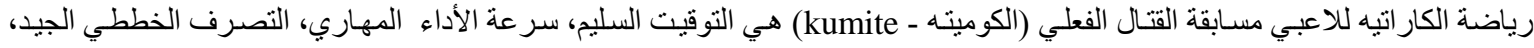

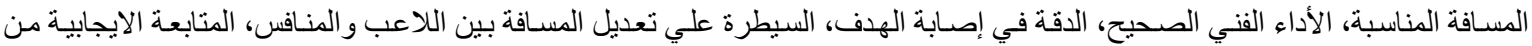

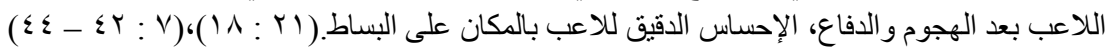

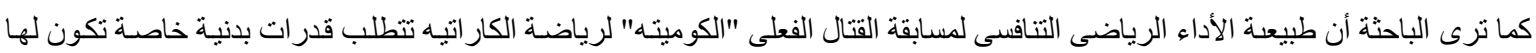

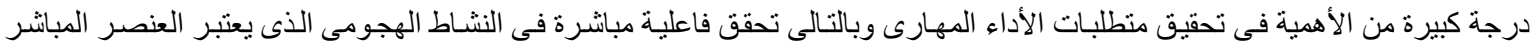

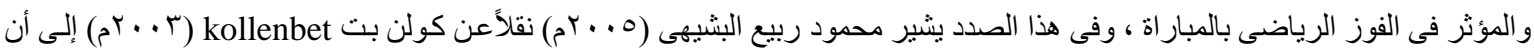

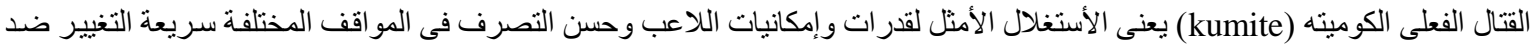

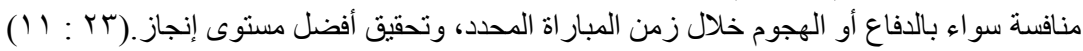

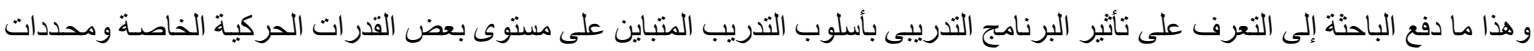

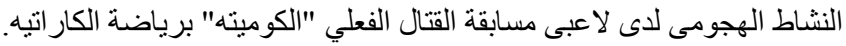

هدف البحث:

يهدف البحث إلى التعرف على تأثير البرنامج التدريبى بأسلوب التدريب المتباين على مستوى بعض القدرات الحركية الخاصـة ومحددات

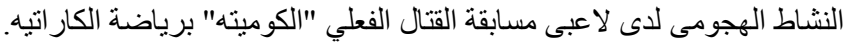

فروض البحث:

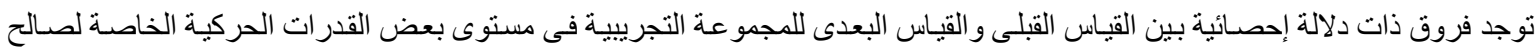
القياس البعدى نتيجة نأثير البرنامج التدريبى بأسلوب التدريب التنباين لاى لاعبى مسابقة القتال الفعلي "الكوميته" برياضية الكار اتيه ؟

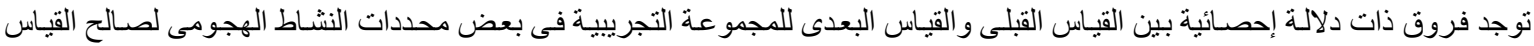
البعدى نتيجة تأثير البرنامج التدريبى بأسلوب التدريب المتباين لدى لاعبى مسابقة القتال الفعلي "الكوميته" برياضية الكار التيه ؟

مصطلحات البحث:

التدريب المتباين: The Contrast training

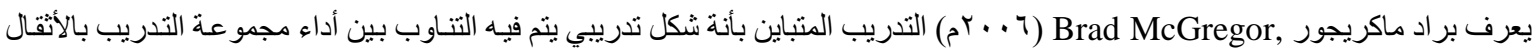

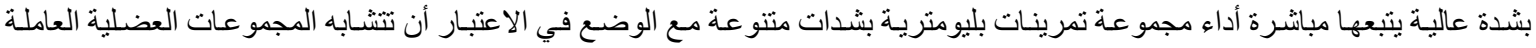

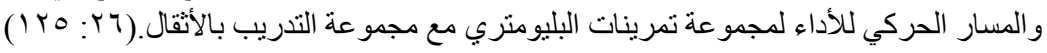

النشاط الهجومى: The attacking activity

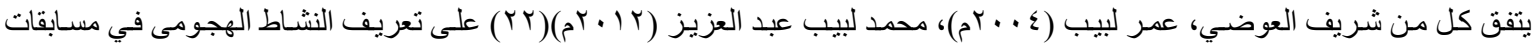

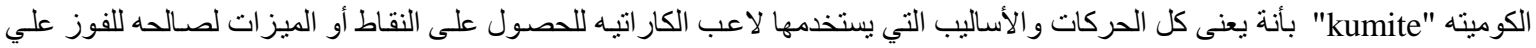

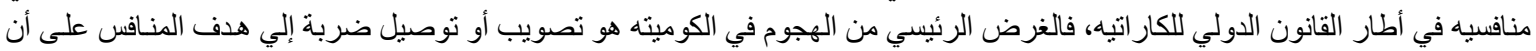

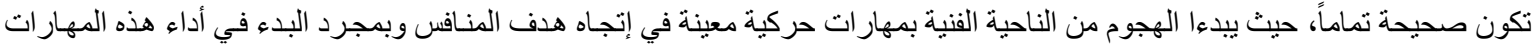

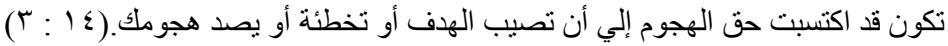

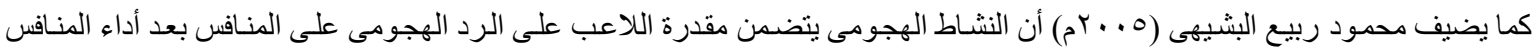

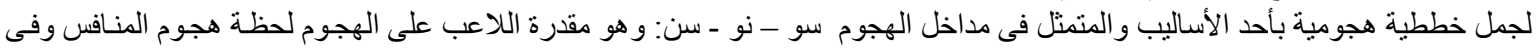

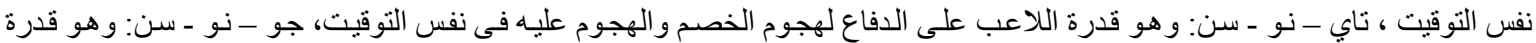

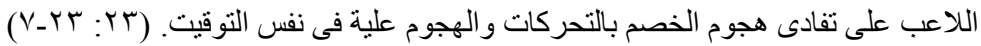


استخدمت الباحثة المنهج التجريبى متبعة التصميم التجريبى ذو القياس القبلى و البعدى لمجموعة تجريبية واحدة، نظر اً لملائمتة لطبيعة البحث.

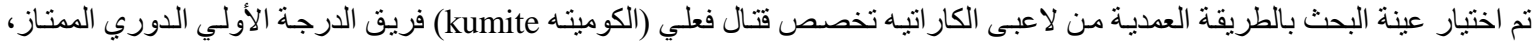

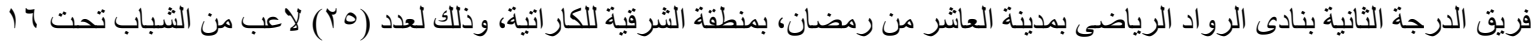

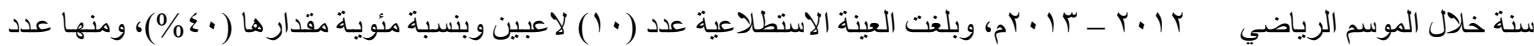

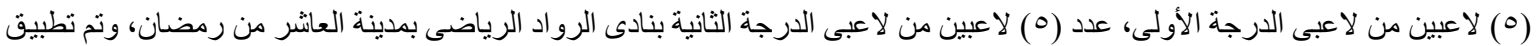

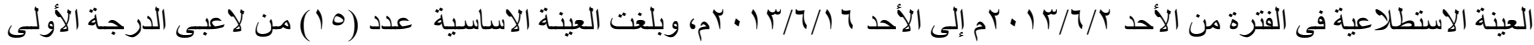

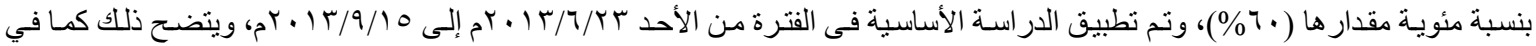

الجدول (1). (1) (1)

\section{جدول (1)}

\section{r. = توصيف عينة البحث}

\begin{tabular}{|c|c|c|c|}
\hline النسبة المئوية & 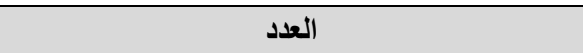 & البيان البيان & م \\
\hline$\% \varepsilon$ & 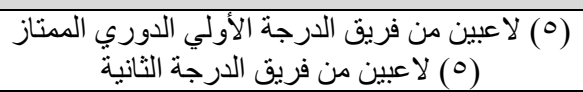 & العينة الاستطلاعية & 1 \\
\hline$\% 4$. & (10) لاعب من فريق الدرجة الأولي الدوري المثتاز & العينة الأساسية & $r$ \\
\hline$\% 1 \ldots$ & (Y0) لاعب من فريق الدرجة الأولي الدوري الممتاز & المجتمع الكلى لعينة البحث & $r$ \\
\hline
\end{tabular}

تجانس عينة البحث:

نم إيجاد التجانس بين أفراد مجتمع البحث فى متغير ات النمو (الطول، الوزن، السن)، والعمر التدريبى و القدرات البدنية الخاصـة، ويتضح ذلك

كما فى جدول (r) (r).

\section{جدول (r) (ات)}

تجانس عينة البحث الكلية (الأساسيةـ الاستطلاعية) فى متغيرات النمو (العن - الطول ـ الوزن) والعمر التدريبى والقدرات البدنية الخاصة $r \cdot=\dot{U}$

\begin{tabular}{|c|c|c|c|c|c|c|c|}
\hline الألتواء & 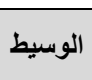 & المعيارى & المسابوسط & القياسة & \multicolumn{2}{|l|}{ 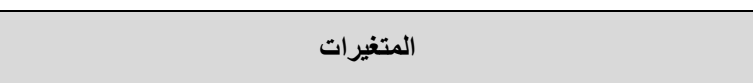 } & s \\
\hline $1, \pi r_{-}$ & 17 & $1,0 \leqslant$ & $10,1 \mathrm{~V}$ & سنة & ال السن & \multirow{3}{*}{$\begin{array}{l}\frac{3}{3} \\
\frac{1}{3} \\
\overline{3}\end{array}$} & \multirow{3}{*}{1} \\
\hline$\cdot, \wedge$ & 17. & $\varepsilon, \wedge \vee$ & $17 ., 15$ & سم & الطول & & \\
\hline$\cdot, \cdot 7$ & TY & 0,99 & $r, 11$ & 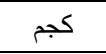 & 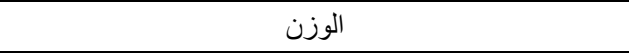 & & \\
\hline$\cdot V V_{-}$ & $\wedge$ & I.Av & V.Or & سنة & \multicolumn{2}{|l|}{ 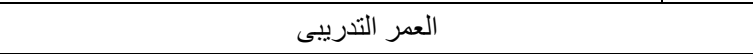 } & r \\
\hline r & $\varepsilon$ & $\cdot .99$ & ๕.) & 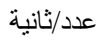 & Gyaku Zuki اختبار اللكمة المستقيمة المعاكسة (بمين) & \multirow{10}{*}{ 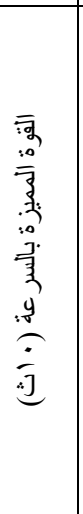 } & \multirow{10}{*}{$r$} \\
\hline$\cdot, 00$ & $\varepsilon$ & 1.0 & ๕.) & عدد/ثانية & اختبار اللكمة المستقيمة المعاكسة (شمال) Gyaku Zuki & & \\
\hline$\cdot .49$ & $\varepsilon$ & 1.4 & $\varepsilon .{ }^{\prime}$ & عدد/ثانية & اختبار اللكمة المستقيمة الأمامية الطولية (يمين) Oi Zuki & & \\
\hline$\cdot . \wedge \wedge$ & $\varepsilon$ & $1 . \cdot r$ & $\varepsilon . \Gamma$ & 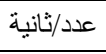 & Oi Zuki اختبار اللكمة المستقيمة الأمامية الطولية (شمال) & & \\
\hline 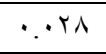 & ๕.0 & 1.9 & $\{.01$ & عدد/ثانية & May- geri اختبار الركلة الأمامية (يمين) & & \\
\hline $.1 \leqslant$ & $\varepsilon$ & 1.1 & $\varepsilon .0$ & 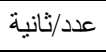 & May- geri اختبار الركلة الأمامية (شمال) & & \\
\hline $1.1 \mathrm{~V}$ & $\varepsilon$ & $1.1 \%$ & $\varepsilon . \leqslant$ & عدد/ثانية & اختبار الركلة الدائرية (يمين) Mawashi- Geri & & \\
\hline $.1 \leqslant$ & $\varepsilon$ & 1.1 & ๕..० & عدد/ثانية & اختبار الركلة الدائرية (شمال) Mawashi- Geri & & \\
\hline$\cdot 9 \cdot$ & $\varepsilon$ & 1. & $\varepsilon . \Gamma$ & 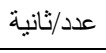 & اختبار الركلة نصف الدائرية (يمين) Ora-mawashi - Geri & & \\
\hline$\because .0 \Lambda_{-}$ & ๕. & $1 . r$ & $\varepsilon, \iota_{\Lambda}$ & 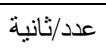 & Ora-mawashi - Geri اختبار الركلة نصف الدائرية (شمال) & & \\
\hline$\cdot r_{0}$ & $\varepsilon .9$ & $\cdot . \wedge \varepsilon$ & $\varepsilon .9 \vee$ & عدد/ثانية & اختبار اللكمة المستقيمة المعاكسة (بمين) Gyaku Zuki & $\overline{3}$ & $\varepsilon$ \\
\hline$\cdot . T \vee \Lambda_{-}$ & 。 & $.9 \vee \pi$ & $\varepsilon . \vee \wedge$ & عدد/ثانية & اختبار اللكمة المستقيمة المعاكسة (شمال) Gyaku Zuki & & \\
\hline
\end{tabular}




\begin{tabular}{|c|c|c|c|c|c|c|c|}
\hline الألتواء معامل & 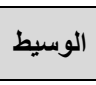 & الألمراف المعيارى & الحستوسط المسبى & والقياس & المتغيرات & & b \\
\hline$\because .0 \varepsilon_{-}$ & $\mathrm{V} . r$ & 1.14 & $\mathrm{v.} \cdot 1$ & عدد/ثانية & Oi Zuki اختبار اللكمة المستقيمة الأمامية الطولية (يمين) & & \\
\hline 1.04 & 7 & 1.1. & 7.07 & عدد/ثانية & Oi Zuki اختبار اللكمة المستقيمة الأمامية الطولية (شمال) & & \\
\hline$\cdot r \wedge$ & 7 & $1 . \mathrm{r}$ & $7.1 \mathrm{~T}$ & عدد/ثانية & May- geri اختبار الركلة الأمامية (يمين) & & \\
\hline 1.1 & 0 & $1 . r_{0}$ & $0 . \leqslant 0$ & عدد/ثانية & May- geri اختبار الركلة الأمامية (شمال) & & \\
\hline$\because \wedge Y_{1}$ & $v_{.} \cdot$ & $1.1 \mathrm{~V}$ & 7.71 & عدد/ثانية & Mawashi- Geri اختبار الركلة الدائرية (يمين) & & \\
\hline $.0 \mathrm{~V}$. & 0 & 1.4 & $0.4 \pi$ & عدد/ثانية & Mawashi- Geri اختبار الركلة الدائرية (شمال) & & \\
\hline$\because 7 \cdot 7$ & 7 & 1.9 & $T . Y$ T & عدد/ثانية & Ora-mawashi - Geri اختبار الركلة نصف الدائرية (يمين) & & \\
\hline 1.19 & 0 & $.9 \vee 9$ & 0.49 & عدد/ثانية & Ora-mawashi - Geri اختبار الركلة نصف الدائرية (شمال) & & \\
\hline$\because \operatorname{tr}$ & $1 \pi .0$ & r. 9. & 15.07 & عدد/ثانية & Gyaku Zuki اختبار اللكمة المستقيمة المعاكسة (يمين) & \multirow{10}{*}{ 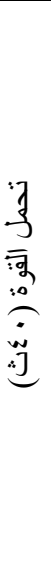 } & \multirow{10}{*}{0} \\
\hline.$V \vee T_{-}$ & it & $1 . Y \wedge$ & $11.7 \mathrm{~V}$ & عدد/ثانية & اختبار اللكمة المستقيمة المعاكسة (شمال) Gyaku Zuki & & \\
\hline 1.01 & IT & r.os & IT.^9 & عدد/ثانية & Oi Zuki اختبار اللكمة المستقيمة الأمامية الطولية (يمين) & & \\
\hline.$\leqslant \vee \tau_{-}$ & 14 & $1.7 \varepsilon$ & IT.VE & عدد/ثانية & Oi Zuki اختبار اللكمة المستقيمة الأمامية الطولية (شمال) & & \\
\hline$\cdot r \wedge r$ & $1 \leq 0$ & T.TV & $1 \leqslant . \wedge \varepsilon$ & عدد/ثانية & May- geri اختبار الركلة الأمامية (يمين) & & \\
\hline . YYS & 14 & r.Y人 & IT.IV & عدد/ثانية & May- geri اختبار الركلة الأمامية (شمال) & & \\
\hline$\cdot Y \cdot T$ & $1 \leq$ & r. $\leqslant \Lambda$ & $1 \leq .1 \mathrm{~V}$ & عدد/ثانية & Mawashi- Geri اختبار الركلة الدائرية (يمين) & & \\
\hline$\cdot 9 \vee V$ & Tr & Y.00 & Tז.הז & عدد/ثانية & Mawashi- Geri اختبار الركلة الدائرية (شمال) & & \\
\hline$\cdot r$ r $_{0}$ & $1 \leq$ & r. 70 & $1 \leq . \Gamma \varepsilon$ & عدد/ثانية & $\begin{array}{c}\text { Ora-mawashi - (ختبار الركلة نصف الدائرية (يمين) } \\
\text { Geri }\end{array}$ & & \\
\hline וسח. & r & 1.99 & r.r. & عدد/ثانية & $\begin{array}{c}\text { Ora-mawashi - اختبار الركلة نصف الدائرية (شمال) } \\
\text { Geri }\end{array}$ & & \\
\hline
\end{tabular}

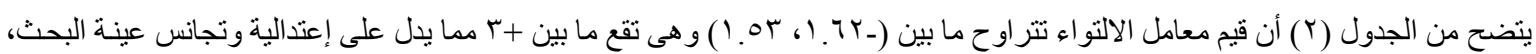
وأنها تقع تحت المنحني الطبيعي. أدوات البحث:

1 - جهاز الرستاميتر قياس الطول لأقرب (سم، ميزان طبى لقياس الوزن لأقرب كجم مرفق (مرفقV).

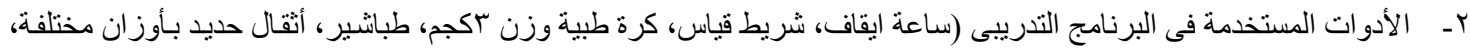

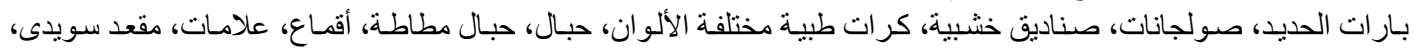
دمبلز، حواجز ،أساتلك مطاطة، جوانتات اكيت أنقال، صفارة).

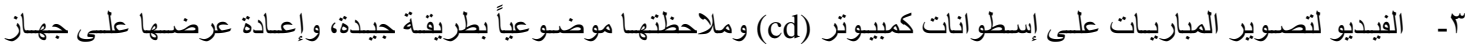
الكمبيوتر لتحليل المباريات. ـ - استمارة تسجيل بيانات اللاعبين في متغير ات النمو (السن - الطول - الوزن) و العمر التدريبى، و أستمارة متغير ات القدرات البدنية الخاصة، و أستمارة محددات النشاط الهجومى (مرفق 9 9)

الأختبارات المستخدمة فى البحث: الأختبارات البذنية:

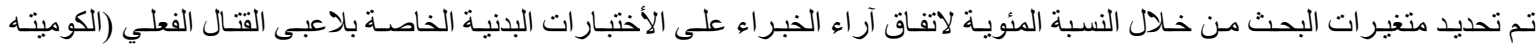

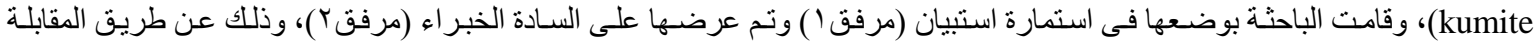

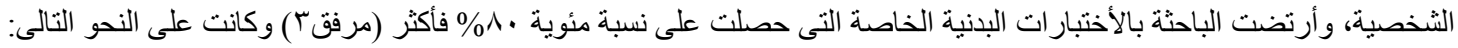
القوة المميزة بالسرعة (• اث) و المنمثلة فى الأختبار ات التالية:

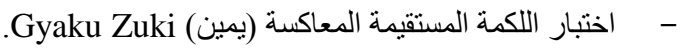
Gyaku Zuki اختبار اللكمة المستقيمة المعاكسة (شمال) - - - 
Oi Zuki اختبار اللكمة المستقيمة الأمامية الطولية مين) -

Oi Zuki اختبار اللكمة المستقيمة الأمامية الطولية (شمال) -

May- geri اختبار الركلة الأمامية (يمين) -

May- geri اختبار الركلة الأمامية (شمال) -

Mawashi- Geri اختبار الركلة الدائرية ميمين) -

Mawashi- Geri اختبار الركلة الدائرية (شمال) -

Ora-mawashi - Geri اختبار الركلة نصف الدائرية (يمين) -

Ora-mawashi - Geri اختبار الركلة نصف الدائرية (شمال) -

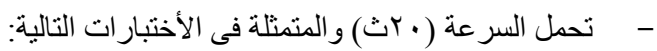

Gyaku Zuki اختبار اللكمة المستقيمة المعاكسة (يمين) -

Gyaku Zuki اختبار اللكمة المستقيمة المعاكسة (شمال) -

Oi Zuki اختبار اللكمة المستقيمة الأمامية الطولية -

Oi Zuki اختبار اللكمة المستقيمة الأمامية الطولية (شمال) -

May- geri اختبار الركلة الأمامية (يمين) -

May- geri اختبار الركلة الأمامية (شمال) -

Mawashi- Geri اختبار الركلة الدائرية (يمين) -

Mawashi- Geri اختبار الركلة الدائرية (شمال) -

Ora-mawashi - Geri اختبار الركلة نصف الدائرية (يمين) -

Ora-mawashi - Geri اختبار الركلة نصف الدائرية (شمال) -

- تحمل القوة ( • عث) و المتمثلة فى الأختبار ات التالية:

Gyaku Zuki اختبار اللكمة المستقيمة المعاكسة (يمين) -

Gyaku Zuki اختبار اللكمة المستقيمة المعاكسة (شمال) -

Oi Zuki اختبار اللكمة المستقيمة الأمامية الطولية (يمين) -

Oi Zuki اختبار اللكمة المستقيمة الأمامية الطولية (شمال) -

May- geri اختبار الركلة الأمامية (يمين) -

May- geri اختبار الركلة الأمامية (شمال) -

Mawashi- Geri اختبار الركلة الدائرية إيمين) -

Mawashi- Geri اختبار الركلة الدائرية (شمال) -

Ora-mawashi - Geri اختبار الركلة نصف الدائرية (يمين) -

Ora-mawashi - Geri اختبار الركلة نصف الدائرية (شمال) -

وترى الباحثة أن هذة الأختبار ات لها معاملات صدق وثبات وموضو عية عاليـة وسبق التأكد من الصدق و الثبـات لها فى العديد من الدراسـات العلمية السابقة.

المتغيرات المهارية: 


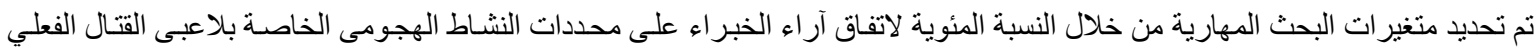

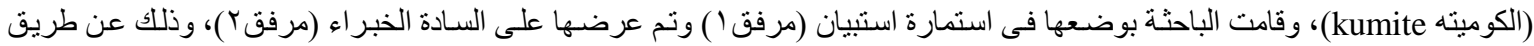

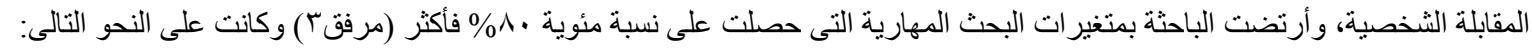


- سو - نو - سن: و هو مقدرة اللاعب على الهجوم لحظة هجوم المنافس وفى نفس التوقيت. - - تاي- نو - سن: وهو قدرة اللاعب على الدفاع لهجوم الخصم و الهجوم عليه فى نفس التوقيت.

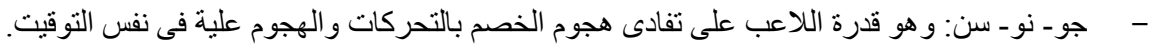

\section{فعالية النشاط الهجومى (بد) و المتمثلة فى:}

- - فعالية النشاط الهجومى فى الدقيقة الأولى من المباراة.

- معالية النشاط الهجومى فى الاقيقة الثانية من المبار اة.

- - فعالية النشاط الهجومى فى الدقيقة الثالثة من المبار اة.

- فعالية النشاط الهجومى فى المجموع الكلي للمبار اة.

البرنامج التتريبي المقترح:

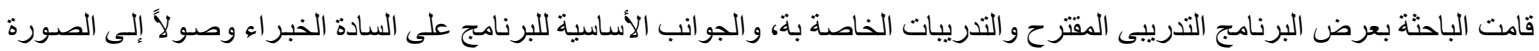

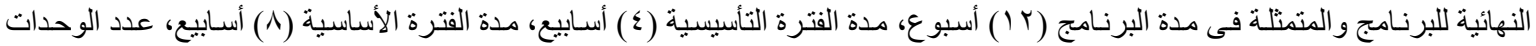

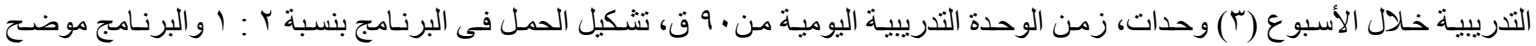

بالتفصيل مرفق (ع). ( )

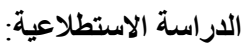

قامت الباحثة بإجر اء الدر اسة الاسنطلاعية على عدد (• (1) لاعبين، منها عدد (0) لاعبين من لاعبى الدرجة الأولى كمجمو عة مميزة، عدد (0)

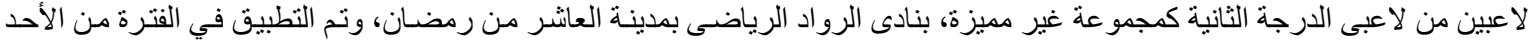

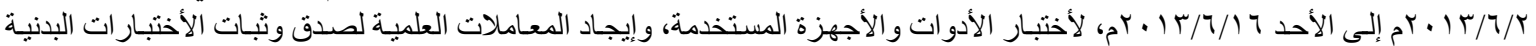

الخاصة، ويتضح ذللك كما فى جدول (rاس، ع ).

المعاملات العلمية للأختبارات البدنية:

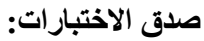

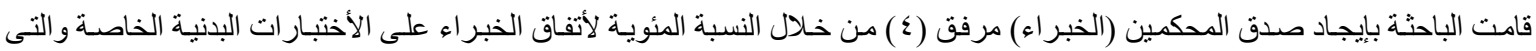

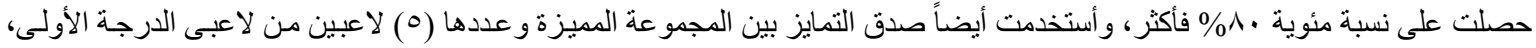

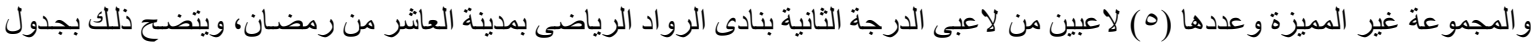

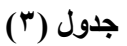

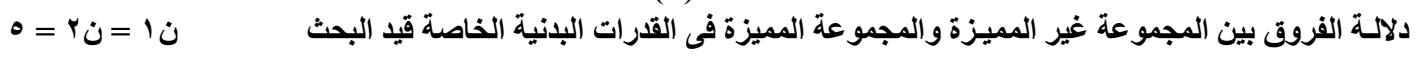

\begin{tabular}{|c|c|c|c|c|c|c|}
\hline \multirow{2}{*}{ قيمة "تلاتها" } & \multicolumn{2}{|c|}{ مجموعة مميزة } & \multicolumn{2}{|c|}{ مجموعة غير مميزة } & \multirow{2}{*}{ الاختبارات البدنية } & \multirow{2}{*}{ البذاتية } \\
\hline & $r \varepsilon$ & $r^{b}$ & $1 \varepsilon$ & $I_{1}$ & & \\
\hline$* r .1 T$ & .99 & $\varepsilon .+Y$ & 1.1 & $r .71$ & اختبار اللكمة المستقيمة المعاكسة (يمين) Gyaku Zuki & \multirow{10}{*}{ (المبزئة } \\
\hline$* r . \mathrm{r}$ & 1.0 & $\varepsilon .1 T$ &.$\wedge 7$ & $r . \leqslant T$ & Gyaku Zuki اختبار اللكمة المستقيمة المعاكسة (شمال) & \\
\hline *trit & 1.4 & E.rT & $1 . r$ & r.l & Oi Zuki اختبار اللكمة المستقيمة الأمامية الطولية (يمين) & \\
\hline$* r . \varepsilon$ & $1 .+r$ & $\varepsilon . \varepsilon$ &.$\wedge \wedge$ & r.01 & Oi Zuki اختبار اللكمة المستقيمة الأمامية الطولية (شمال) & \\
\hline$* r .90$ & 1.9 & $\varepsilon .0$ & .199 & r.^9 & اختبار الركلة الأمامية (يمين) May- geri & \\
\hline$* r . \leqslant 0$ & 1.1 & $\varepsilon . r$ & $\cdot V Y$ & r.70 & May- geri اختبار الركلة الأمامية (شمال) & \\
\hline *Y.Ar & 1.14 & $\varepsilon . \varepsilon r$ & $\because V \mu$ & ז.ru & اختبار الركلة الدائرية (يمين) Mawashi- Geri & \\
\hline$* Y .97$ & 1.1 & $\varepsilon .1 \leqslant$ & $\cdot r l$ & T.YT & اختبار الركلة الدائرية (شمال) Mawashi- Geri & \\
\hline$* 4.19$ & 1. & 5.10 & $\cdot 199$ & r.^^ & Ora-mawashi - Geri اختبار الركلة نصف الدائرية (يمين) & \\
\hline$* \Gamma$. AV & 1.4 & $\leqslant . T_{0}$ & $.9 V$ & r.rq & Ora-mawashi - Geri اختبار الركلة نصف الدائرية (شمال) & \\
\hline
\end{tabular}




\begin{tabular}{|c|c|c|c|c|c|c|}
\hline \multirow{2}{*}{ قيمة "تلاتلها } & \multicolumn{2}{|c|}{ مجموعة مميزة } & \multicolumn{2}{|c|}{ مجموعة غير مميزة } & \multirow{2}{*}{ الاختبارات البدنية } & \multirow{2}{*}{ الصفاتية } \\
\hline & $r \varepsilon$ & $r^{\beta}$ & $1 \varepsilon$ & $\log ^{2}$ & & \\
\hline$* Y . Y V$ & $\cdot \wedge \uparrow$ & 0.99 & $1 . \mu \varepsilon$ & $\varepsilon .9$ & Gyaku Zuki اختبار اللكمة المستقيمة المعاكسة (يمين) & \multirow{10}{*}{ السرعل } \\
\hline$* Y$. $\leqslant \wedge$ & $\because 9 \times 1$ & 0.10 & 1.17 & $\leqslant . \leqslant 7$ & Gyaku Zuki اختبار اللكمة المستقيمة المعاكسة (شمال) & \\
\hline$* \varepsilon .1 \wedge$ & 1.1 & 7.11 & 1. ro & $\varepsilon .91$ & Oi Zuki اختبار اللكمة المستقيمة الأمامية الطولية (يمين) & \\
\hline$* \varepsilon .1 T$ & 1.1 & 7.04 & $1 . \wedge 1$ & $\varepsilon .1$ & Oi Zuki اختبار اللكمة المستقيمة الأمامية الطولية (شمال) & \\
\hline$* \leqslant .90$ & 1.4 & 7.9 & $\because \vee q$ & $\varepsilon .19$ & May- geri اختبار الركلة الأمامية (يمين) & \\
\hline$* Y . \leqslant 1$ & 1.11 & $0 . \leqslant Y$ & $.7 V$ & $\leqslant .10$ & May- geri اختبار الركلة الأمامية (شمال) & \\
\hline$* \varepsilon . \wedge r$ & $1.1 \mathrm{~V}$ & 7.71 & I.r. & $\varepsilon . \leqslant V$ & Mawashi- Geri اختبار الركلة الدائرية (يمين) & \\
\hline$* Y .97$ & 1.19 & $0 . r 4$ & $\because \varepsilon V$ & $\varepsilon .+r$ & Mawashi- Geri اختبار الركلة الدائرية (شمال) & \\
\hline$*$ *IV & 1.9 & T.Y & 1.19 & $\varepsilon . r \varepsilon$ & Ora-mawashi - Geri اختبار الركلة نصف الدائرية (يمين) & \\
\hline$* 111$ & $.9 V$ & 0.99 &.$\wedge 9$ & $\varepsilon . \varepsilon$ & Ora-mawashi - Geri اختبار الركلة نصف الدائرية (شمال) & \\
\hline$* r$ r. & r.AV & IT.OT & 1.19 & $1 \cdot . r 7$ & Gyaku Zuki اختبار اللكمة المستقيمة المعاكسة (يمين) & \multirow{10}{*}{ تحمل } \\
\hline$* r .99$ & $1 . Y \leq$ & 11.74 & 1.1 & $\wedge .1 \mathrm{~V}$ & Gyaku Zuki اختبار اللكمة المستقيمة المعاكسة (شمال) & \\
\hline *r.re & r. $\leqslant 9$ & IY.Vq & $1 . \varepsilon$ & 9.19 & Oi Zuki اختبار اللكمة المستقيمة الأمامية الطولية (يمين) & \\
\hline$* Y .0 \leqslant$ & 1.07 & 19.79 & .99 & $1 \cdot .70$ & Oi Zuki اختبار اللكمة المستقيمة الأمامية الطولية (شمال) & \\
\hline$* r .00$ & 1.09 & $1 \leq . \vee \wedge$ & T.YV & $11 . v \varepsilon$ & May- geri اختبار الركلة الأمامية (يمين) & \\
\hline$* 4.09$ & r. 19 & 11.0 & $r . r$ & $1 . .1 \mathrm{~V}$ & May- geri اختبار الركلة الأمامية (شمال) & \\
\hline$* r .9$ & T. Tq & $1 \leq .11$ & 1.4 & 11.1 & Mawashi- Geri اختبار الركلة الدائرية (يمين) & \\
\hline$*$ *r. $r \varepsilon$ & $r . \leqslant 1$ & IT.VA & r.11 & $1 \cdot . \wedge r$ & Mawashi- Geri اختبار الركلة الدائرية (شمال) & \\
\hline$* \varepsilon, Y Y$ & r.OV & $1 \leqslant .49$ & I.AV & $9.0 \leq$ & Ora-mawashi - Geri اختبار الركلة نصف الدائرية (يمين) & \\
\hline$* \varepsilon .10$ & $1 . \wedge \wedge$ & 11.11 & .99 & 9.1 & Ora-mawashi - Geri اختبار الركلة نصف الدائرية (شمال) & \\
\hline
\end{tabular}

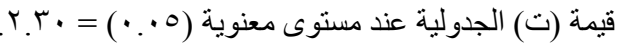

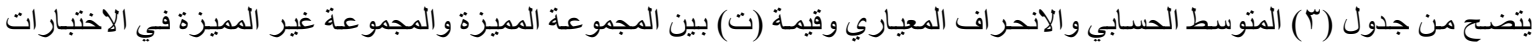

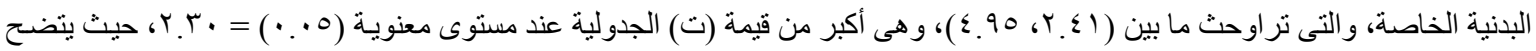

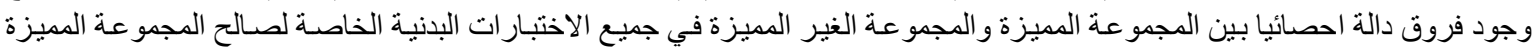
الأمر الذي يشير الي صدق الاختبارات فيما وضعت لقياسة.

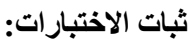

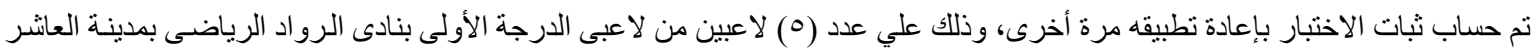

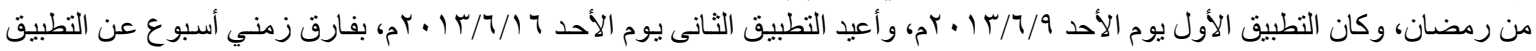
الأول، حيث طبقت نفس الاختبار ات وبنفس الأدوات و الظروف، ويتضح ذلك بجدول (ع ).

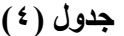

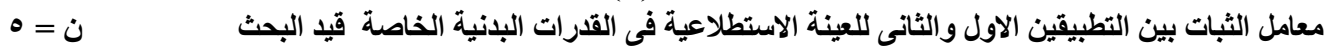

\begin{tabular}{|c|c|c|c|c|c|c|}
\hline \multirow{2}{*}{ 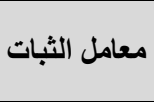 } & \multicolumn{2}{|c|}{ 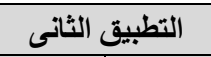 } & \multicolumn{2}{|c|}{ التطبيق الاول } & \multirow{2}{*}{ الاختبارات البدنية } & \multirow{2}{*}{ البذنية } \\
\hline & ع & $t^{b}$ & $1 \varepsilon$ & م & & \\
\hline$* .9 r r$ & $.9 V$ & $\varepsilon .$. & .99 & $\varepsilon . r$ & Gyaku Zuki اختبار اللكمة المستقيمة المعاكسة (يمين) & \multirow{7}{*}{ المبرة القوة } \\
\hline$* .9 .9$ & 1.1 & £.11 & 1.0 & $\varepsilon .1 T$ & اختبار اللكمة المستقيمة المعاكسة (شمال) Gyaku Zuki & \\
\hline$* .9 \vee r$ & $1 . \cdots$ & $\varepsilon, r$ & 1.4 & $\varepsilon . r \mu$ & Oi Zuki اختبار اللكمة المستقيمة الأمامية الطولية (يمين) & \\
\hline$* .911$ & $1 . \cdot \varepsilon$ & $\varepsilon .0$ & 1.4 & $\varepsilon$ & Oi Zuki اختبار اللكمة المستقيمة الأمامية الطولية (شمال) & \\
\hline$* .911$ & 1.1 & $\varepsilon .7$ & 1.99 & $\varepsilon .0$ & اختبار الركلة الأمامية (يمين) May- geri & \\
\hline$* . .9 \times 1$ & $1 . \varepsilon$ & $\varepsilon . r$ & 1.1 & $\varepsilon . r$ & May- geri اختبار الركلة الأمامية (شمال) & \\
\hline$* .974$ & 1.1. & $\varepsilon$. & 1.15 & $\varepsilon$ \& & Mawashi- Geri اختبار الركلة الدائرية (يمين) & \\
\hline
\end{tabular}




\begin{tabular}{|c|c|c|c|c|c|c|}
\hline \multirow{2}{*}{ 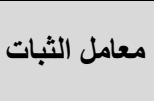 } & \multicolumn{2}{|c|}{ التطبيق الثانى } & \multicolumn{2}{|c|}{ 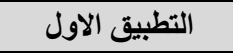 } & \multirow{2}{*}{ الاختبارات البذنية } & \multirow{2}{*}{ البدنية } \\
\hline & $r \varepsilon$ & $t^{p}$ & $1 \varepsilon$ & $l_{1}$ & & \\
\hline$* . .9 \cdot v$ & 1.11 & $\varepsilon .14$ & 1.1 & $\varepsilon .1 \leqslant$ & Mawashi- Geri اختبار الركلة الدائرية (شمال) & \\
\hline$* .9 \leq r$ & $1 . r$ & $\varepsilon .1 T$ & 1. & $\varepsilon .10$ & Ora-mawashi - Geri اختبار الركلة نصف الدائرية (يمين) & \\
\hline$* .91$ & $1 \cdot \varepsilon$ & $\varepsilon . \Gamma$ & 1.4 & 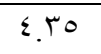 & Ora-mawashi - Geri اختبار الركلة نصف الدائرية (شمال) & \\
\hline$* .900$ &.$\wedge r$ & 0.97 &.$\wedge 7$ & 0.99 & Gyaku Zuki اختبار اللكمة المستقيمة المعاكسة (يمين) & \multirow{10}{*}{ السرعة } \\
\hline$* .9+9$ & .97 & $0 . V Y$ & $.9 \vee 1$ & 0.10 & Gyaku Zuki اختبار اللكمة المستقيمة المعاكسة (شمال) & \\
\hline$* .907$ & 1.14 & 7.11 & 1.1 & 7.11 & Oi Zuki اختبار اللكمة المستقيمة الأمامية الطولية (يمين) & \\
\hline *. & 1.11 & $7.0 \leq$ & 1.1. & $7.0 Y$ & Oi Zuki اختبار اللكمة المستقيمة الأمامية الطولية (شمال) & \\
\hline$* .971$ & 1.19 & ᄀ.. & 1.1 & 7.9 & May- geri اختبار الركلة الأمامية (يمين) & \\
\hline$* .949$ & 1.11 & 0.19 & 1.41 & $0 . \leqslant Y$ & May- geri اختبار الركلة الأمامية (شمال) & \\
\hline$* .919$ & 1.19 & 7.79 & $1.1 \mathrm{~V}$ & 7.71 & Mawashi- Geri اختبار الركلة الدائرية (يمين) & \\
\hline$* .909$ & $1.1 \mathrm{~V}$ & $0 . r$. & 1.19 & $0 . Y Y$ & Mawashi- Geri اختبار الركلة الدائرية (شمال) & \\
\hline$* .9 \vee \leqslant$ & 1.1 & 7.41 & 1.9 & T. Y & Ora-mawashi - Geri اختبار الركلة نصف الدائرية (يمين) & \\
\hline$* .97 \pi$ & .91 & $0.9 V$ & $.9 V$ & 0.99 & Ora-mawashi - Geri اختبار الركلة نصف الدائرية (شمال) & \\
\hline$* .91 \leq$ & r.AT & $1 T .04$ & Y.AV & IT.OT & Gyaku Zuki اختبار اللكمة المستقيمة المعاكسة (يمين) & \multirow{10}{*}{ (تحمل القوة } \\
\hline$* .9 r V$ & $1 . r o$ & 11.70 & $1 . Y \leqslant$ & 11.74 & Gyaku Zuki اختبار اللكمة المستقيمة المعاكسة (شمال) & \\
\hline$* .9 \wedge r$ & $Y . \leqslant T$ & IT.VA & r. $\leqslant 9$ & IY.V9 & Oi Zuki اختبار اللكمة المستقيمة الأمامية الطولية (يمين) & \\
\hline$* .90 \mathrm{~V}$ & 1.01 & IT.V & 1.07 & 14.79 & Oi Zuki اختبار اللكمة المستقيمة الأمامية الطولية (شمال) & \\
\hline$* .9 \mu$ & Y. & $1 \leq . \vee 9$ & r.09 & $1 \leq . \vee \wedge$ & May- geri اختبار الركلة الأمامية (يمين) & \\
\hline$* .970$ & 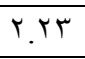 & IT.r & r.19 & 11.0 & May- geri اختبار الركلة الأمامية (شمال) & \\
\hline$* .9 \wedge \leq$ & $r \leqslant r$ & $1 \leq .1 \leq$ & r. & $1 \leq .11$ & Mawashi- Geri اختبار الركلة الدائرية (يمين) & \\
\hline$* .977$ & r.01 & $1 \pi . \wedge$. & $r . \leqslant \Lambda$ & IT.VA & Mawashi- Geri اختبار الركلة الدائرية (شمال) & \\
\hline$* .94$ & r.or & $1 \leq . Y V$ & r.OV & $1 \varepsilon .49$ & Ora-mawashi - Geri اختبار الركلة نصف الدائرية (يمين) & \\
\hline$* .911$ & 1.10 & 11.17 & $1 . \wedge \wedge$ & 11.11 & Ora-mawashi - Geri اختبار الركلة نصف الدائرية (شمال) & \\
\hline
\end{tabular}

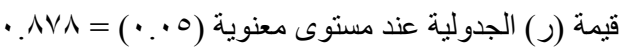

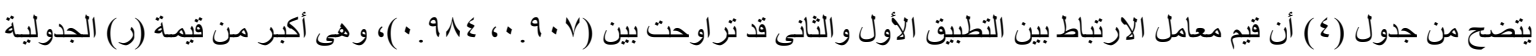

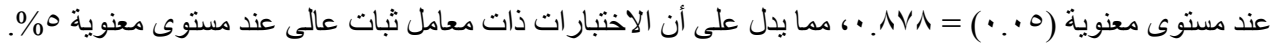

\section{المعاملات العلمية للاستمارة تحليل المباريات فى محددات النشاط الهجومى:}

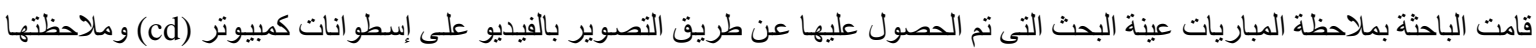

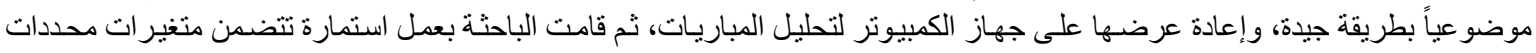

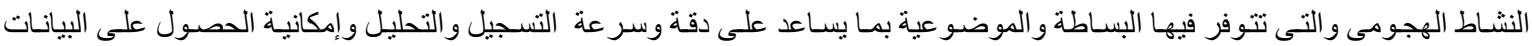

المطلوبة.

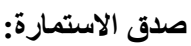

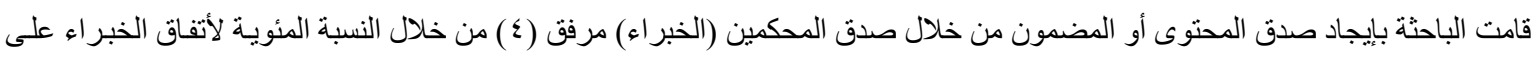

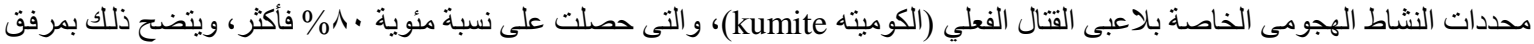

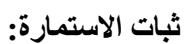

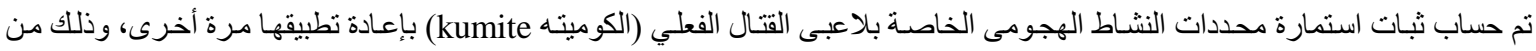

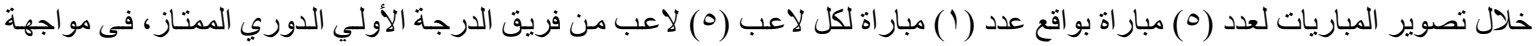

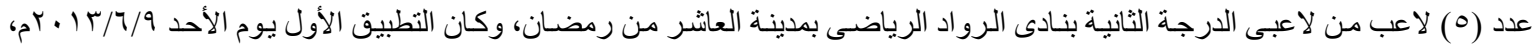




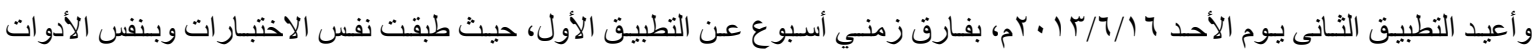
و الظروف، ويتضح ذلك بجدول (0).

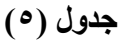

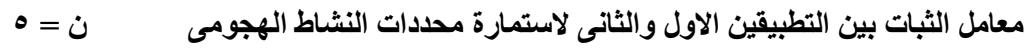

\begin{tabular}{|c|c|c|c|c|c|c|}
\hline \multirow{2}{*}{ 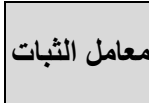 } & \multicolumn{2}{|c|}{ التطبيق الثانى } & \multicolumn{2}{|c|}{ التطبيق الاول } & \multirow{2}{*}{\multicolumn{2}{|c|}{ محدات النشاط الهجومى }} \\
\hline & $r \varepsilon$ & $t^{p}$ & $\varepsilon$ & ק & & \\
\hline$* 1$. & $r . \varepsilon r$ & $\vee . \wedge 1$ & T. $\leqslant T$ & $\checkmark . \wedge 1$ & مداخل الهجوم سو - نو - سن & \multirow{3}{*}{ مداخل } \\
\hline$* .999$ & r.17 & r.71 & T.17 & $r .71$ & مداخل الهجوم تاي- نو - سن & \\
\hline$* 1$. & 1.01 & 1.87 & 1.01 & 1.17 & مداخل الهجوم جو - نو - سن & \\
\hline$* 1$. & 1.21 & $r .01$ & 1.81 & $r .01$ & فعالية النشاط الهجومى فى الدقيقة الأولى من المباراة & \multirow{4}{*}{ 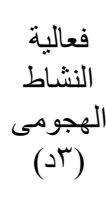 } \\
\hline$* 1$. & 1.20 & $r . \leqslant \Lambda$ & $1 . \leqslant 0$ & $r . \leqslant \wedge$ & فعالية النشاط الهجومى فى الدقيقة الثانية من المبار اة & \\
\hline$* 1$. & $1 . r 1$ & r.Ar & 1.4 & Y.Ar & فعالية النشاط الهجومى فى الدقيقة الثالثة من المبار اة & \\
\hline$* 1$. & r.£ & $9 . \wedge 1$ & Y. $\leqslant 1$ & $9 . \wedge 1$ & فعالية النشاط الهجومى فى المجموع الكلي للمبار اة & \\
\hline
\end{tabular}

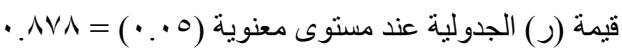

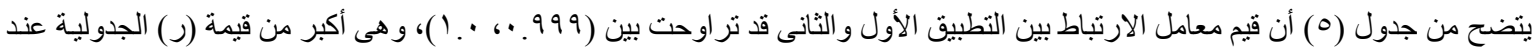

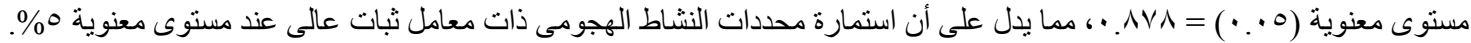

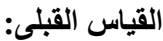

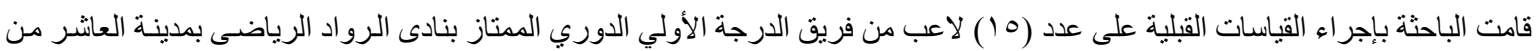

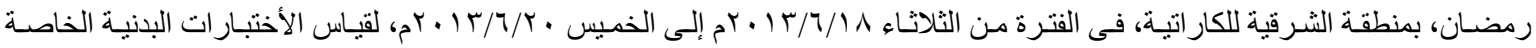

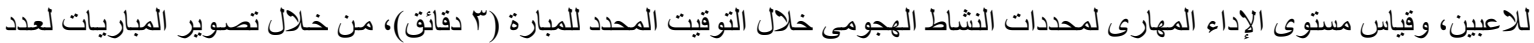

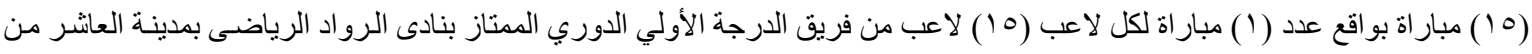

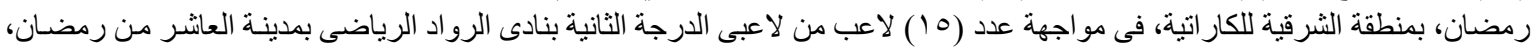

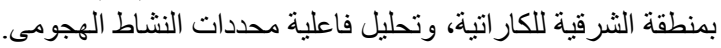

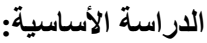

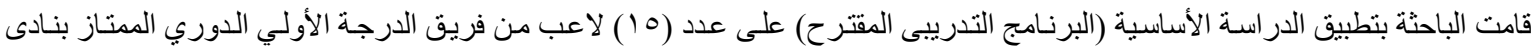

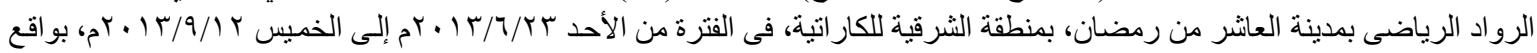
(1) (1) ( أسبو عاً.

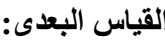

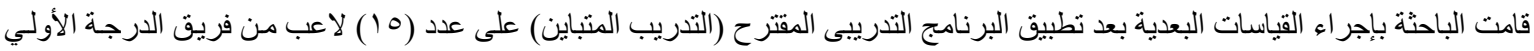

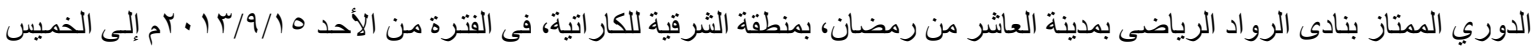

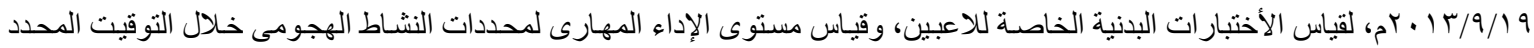

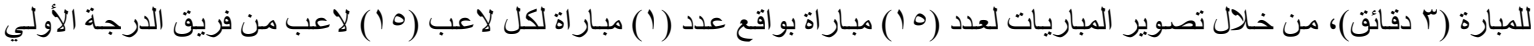

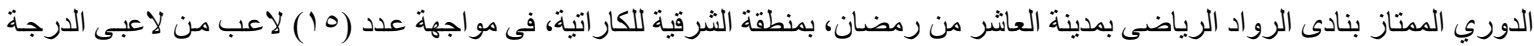

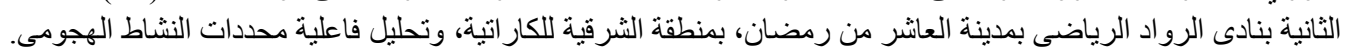

المعالجات الإحصائية:

تم استخدام المعالجات الإحصائية المناسبة لطبيعة البحث، بإستخدام برنامج (10) SPSS لإجر اء العمليات الإحصائية للبحث، و المنمثلة فى:

Arithmtic Mean

المتوسط الحسابى

Median

الوسيط 
Standard Deviastion

Coefficient of Skewness

The Percent

T. Test

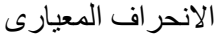

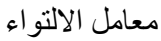

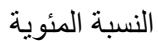

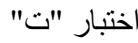

عرض النتائج ومناقشتها:

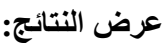

\section{جدول (•)}

المتوسطات الحسابية والأنحراف المعيارى ودلالة الفروق (قيمة "ت") بين القياس القبلى والقياس البعدى للمجموعة التجريبية فى القدرات

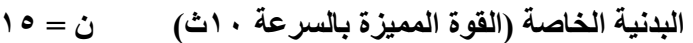

\begin{tabular}{|c|c|c|c|c|c|c|}
\hline \multirow{2}{*}{ قودلالتها "ت" } & \multicolumn{2}{|c|}{ القياس البعدى } & \multicolumn{2}{|c|}{ القياس القبلى } & \multirow{2}{*}{ الاختبارات البدنية } & \multirow{2}{*}{ البذراتي } \\
\hline & $r \varepsilon$ & $r_{p}$ & $1 \varepsilon$ & م & & \\
\hline$* 7.97$ &..$V Y$ & $\Lambda . \Gamma \varepsilon$ & $1 . \leqslant r$ & $\varepsilon .77$ & Gyaku Zuki اختبار اللكمة المستقيمة المعاكسة (يمين) & \multirow{10}{*}{ (المميزة } \\
\hline$* \vee .09$ & 1.1 & ^.) & $1 . r$ & $\varepsilon . r$ & اختبار اللكمة المستقيمة المعاكسة (شمال) Gyaku Zuki & \\
\hline$* 0 . \wedge$. & $1 . \leqslant 0$ & ^. $\leqslant \varepsilon$ & $1 . \varepsilon r$ & $\varepsilon .07$ & Oi Zuki اختبار اللكمة المستقيمة الأمامية الطولية (يمين) & \\
\hline$* \lambda_{.} \cdot 1$ &. $\mathrm{VA}$ & ᄉ.). & $1.1 Y$ & $\varepsilon . \mu q$ & Oi Zuki اختبار اللكمة المستقيمة الأمامية الطولية (شمال) & \\
\hline$* \vee .70$ & $\cdot .11$ & A.ro & $1 . r$. & $\varepsilon . \vee \wedge$ & اختبار الركلة الأمامية (يمين) May- geri & \\
\hline$* 0.01$ & $1 . r$ & $\Lambda .14$ & $1 . \varepsilon$ & $\varepsilon . \vee 9$ & اختبار الركلة الأمامية (شمال) May- geri & \\
\hline$* 0 . \mathrm{VV}$ & 1.9 & $\vee .99$ & $1 . \leqslant 1$ & $\varepsilon .7 V$ & اختبار الركلة الدائرية (يمين) Mawashi- Geri & \\
\hline$* \vee .00$ & 1.1 & $\vee . \varepsilon \varepsilon$ & $\cdot \mathrm{V} \varepsilon$ & $\varepsilon . \mu$ & اختبار الركلة الدائرية (شمال) Mawashi- Geri & \\
\hline$*\rceil . \wedge r$ & 1.0 & $\Lambda . r$ & $1.1 \mathrm{~V}$ & $\varepsilon . \vee \varepsilon$ & اختبار الركلة نصف الدائرية (يمين) Ora-mawashi - Geri & \\
\hline$* 4.01$ & $1 . \varepsilon$ & ᄉ. . & $1 . \varepsilon \cdot$ & $\varepsilon .0 \mathrm{~T}$ & Ora-mawashi - Geri اختبار الركلة نصف الدائرية (شمال) & \\
\hline
\end{tabular}

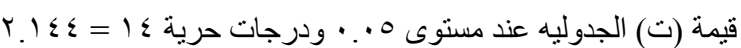

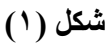

يوضح المتوسطات الحسابية بين القياس القبلى والبعدي للمجموعة التجريبية فى القدرات البذنية الخاصة (القوة المميزة بالسرعة ـ اث)

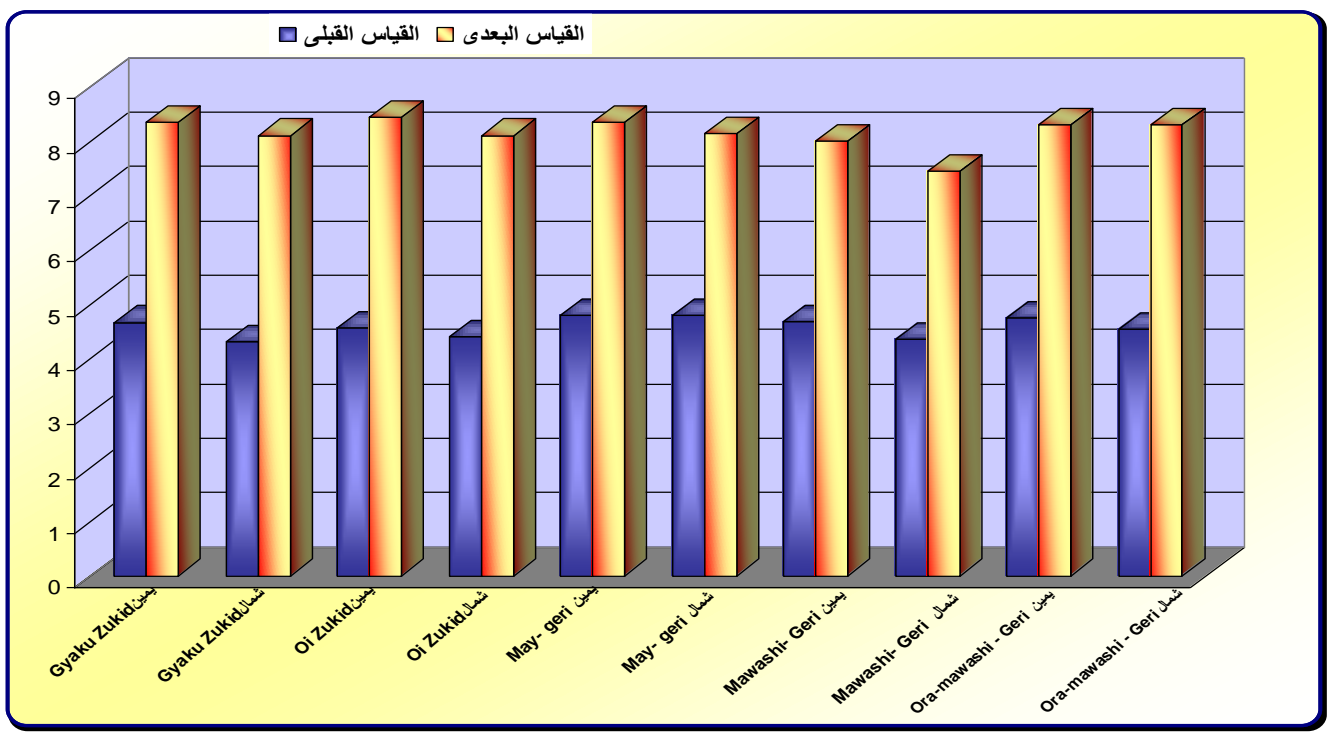




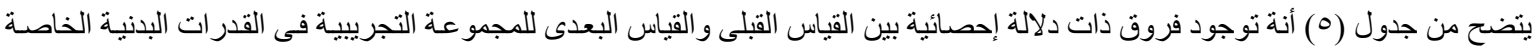

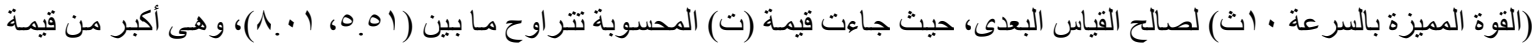

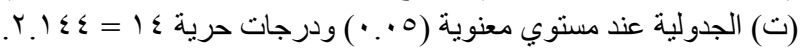

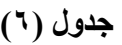

المتوسطات الحسابية والأنحراف المعيارى ودلالة الفروق (قيمة "ت") بين القياس القبلى والقياس البعدى للمجموعة التجريبية فى القدرات

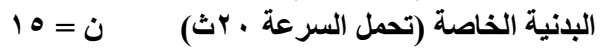

\begin{tabular}{|c|c|c|c|c|c|c|}
\hline \multirow{2}{*}{ قيمة "تلتها" } & \multicolumn{2}{|c|}{ القياس البعدى } & \multicolumn{2}{|c|}{ 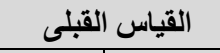 } & \multirow{2}{*}{ الاختبارات البانية } & \multirow{2}{*}{ البذنية } \\
\hline & $r \varepsilon$ & $r_{p}$ & $\varepsilon$ & ما & & \\
\hline$* \wedge . \wedge r$ & $1 . r$ & 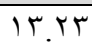 & 1.01 & $7 . \S 1$ & Gyaku Zuki اختبار اللكمة المستقيمة المعاكسة (يمين) & \multirow{10}{*}{ 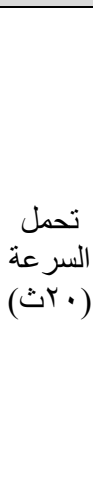 } \\
\hline$* 9.1$. & 1.17 & $1 \% . \leqslant 7$ & 1.04 & $0 . \leqslant r$ & Gyaku Zuki اختبار اللكمة المستقيمة المعاكسة (شمال) & \\
\hline$* 1 \cdot .14$ & $1 . \leqslant 0$ & $1 \leq .14$ & $1 . \cdot \varepsilon$ & $7 . r T$ & Oi Zuki اختبار اللكمة المستقيمة الأمامية الطولية (يمين) & \\
\hline$* 9.9$. & 1.04 & $1 \% .71$ & $1 . r$ & 7.19 & Oi Zuki اختبار اللكمة المستقيمة الأمامية الطولية (شمال) & \\
\hline$* \wedge .11$ & 1.10 & IT.AV & 1.01 & $\begin{array}{r}. T 4 \\
\end{array}$ & May- geri اختبار الركلة الأمامية (يمين) & \\
\hline$* \Lambda . \vee \vee$ & T..0 & Tr.rT & $1 . Y V$ & $\mathrm{~V} .10$ & May- geri اختبار الركلة الأمامية (شمال) & \\
\hline$* Y . \wedge r$ & 1.04 & 115.07 & $1 . v 1$ & V.os & اختبار الركلة الدائرية (يمين) Mawashi- Geri & \\
\hline$* V . \leqslant 7$ & $1 . \leqslant 1$ & $1 \% . \leqslant 0$ & $1 . r 1$ & V.YT & Mawashi- Geri اختبار الركلة الدائرية (شمال) & \\
\hline$* Y$. $\leqslant Y$ & $1 . \leqslant 9$ & $1 T .0 \mathrm{~V}$ & $1 . \varepsilon$ & $\mathrm{V.11}$ & اختبار الركلة نصف الدائرية (يمين) Ora-mawashi - Geri & \\
\hline$* \nu . r \Lambda$ & $1 . \varepsilon$ & 15.49 & $.9 \vee 9$ & V.11 & Ora-mawashi - Geri اختبار الركلة نصف الدائرية (شمال) & \\
\hline
\end{tabular}

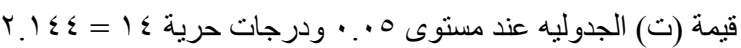

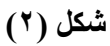

يوضح المتوسطات الحسابية بين القياس القبلى والبعدي للمجموعة التجريبية فى القدرات البدنية الخاصة (تحمل السرعة . ب ث)

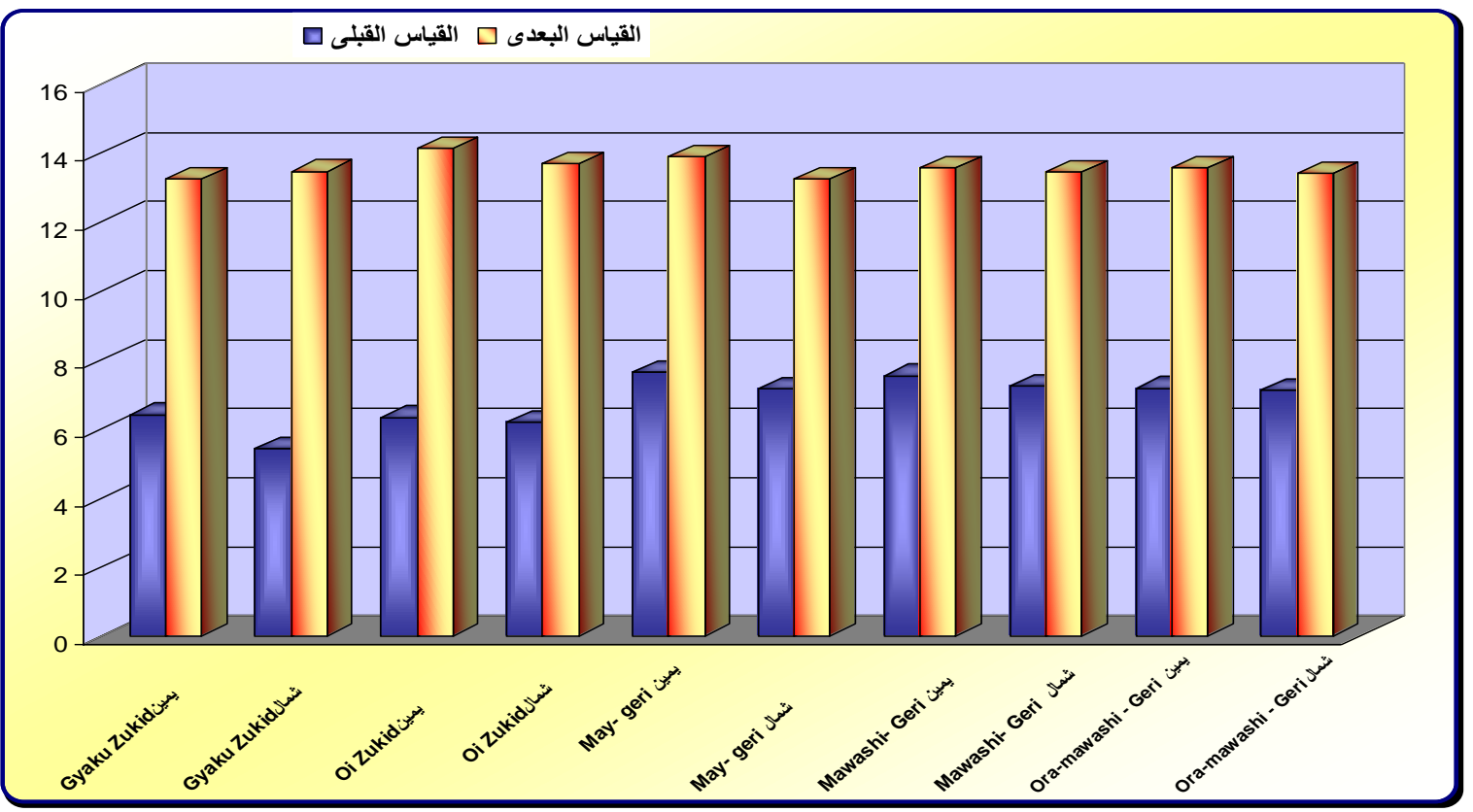

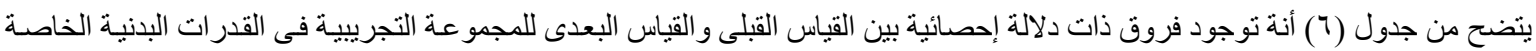

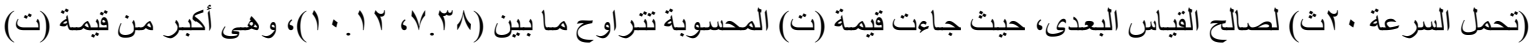

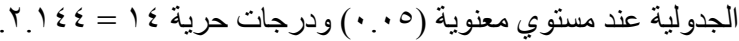




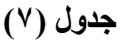

المتوسطات الحسابية والأنحراف المعيارى ودلالة الفروق (قيمة "ت") بين القياس القبلى والقياس البعدى للمجموعة التجريبية فى القدرات

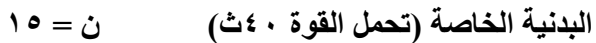

\begin{tabular}{|c|c|c|c|c|c|c|}
\hline \multirow{2}{*}{ قيمة "تلاتها" } & \multicolumn{2}{|c|}{ القياس البعدى } & \multicolumn{2}{|c|}{ القياس القبلى } & \multirow{2}{*}{ الاختبارات البذنية } & \multirow{2}{*}{ القبرات البذنية } \\
\hline & $r \varepsilon$ & $r_{p}$ & $1 \varepsilon$ & ما & & \\
\hline$* \uparrow . \wedge 1$ & $1 . V \varepsilon$ & YI.TV & r.10 & $1 \leqslant .11$ & اختبار اللكمة المستقيمة المعاكسة (يمين) Gyaku Zuki & \multirow{10}{*}{ لحمل القوة } \\
\hline$* 7 . \leqslant \leq$ & $1 . \mathrm{rA}$ & TI.rV & $Y . \cdot 7$ & $1 T . T V$ & اختبار اللكمة المستقيمة المعاكسة (شمال) Gyaku Zuki & \\
\hline$* 7.00$ & $1 . r \cdot$ & YI.A9 & 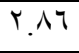 & $1 \leq .11$ & Oتخبار اللكمة المستقيمة الأمامية الطولية (يمين) Oi Zuki & \\
\hline *I.YT & 1.71 & $r \cdot . \wedge 9$ & 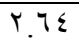 & $1 \Gamma .99$ & Oi Zuki اختبار اللكمة المستقيمة الأمامية الطولية (شمال) & \\
\hline$* 0.09$ & T.TI & Y1.09 & r.VA & 10.1 & May- geri اختبار الركلة الأمامية (يمين) & \\
\hline$* 0 . \leqslant 7$ & r.Or & $r ! . \leqslant V$ & r.10 & $1 \leq .94$ & May- geri اختبار الركلة الأمامية (شمال) & \\
\hline$* 0.97$ & r.AI & Y1.07 & r.90 & $1 \leq .91$ & Mawashi- Geri اختبار الركلة الدائرية (يمين) & \\
\hline$* 0.97$ & $r . \wedge 1$ & $Y 1.07$ & r.90 & $1 \leq . \wedge 1$ & Mawashi- Geri اختبار الركلة الدائرية (شمال) & \\
\hline$* Y . Y T$ & r.10 & $r \cdot .7 V$ & T.70 & $1 \leqslant . \Gamma \leqslant$ & اختبار الركلة نصف الدائرية (يمين) Ora-mawashi - Geri & \\
\hline$* \vee .1 \wedge$ & T.VV & $r \cdot r r$ & $r .1$ & $1 T . r \leq$ & Ora-mawashi - Geri اختبار الركلة نصف الدائرية (شمال) & \\
\hline
\end{tabular}

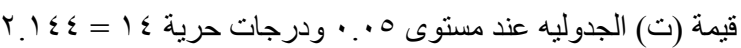

شكل (ب) (ب)

يوضح المتوسطات الحسابية بين القياس القبلى والبعدي للمجموعة التجريبية فى القدرات البدنية الخاصة (تحمل القوة • ؛ث)

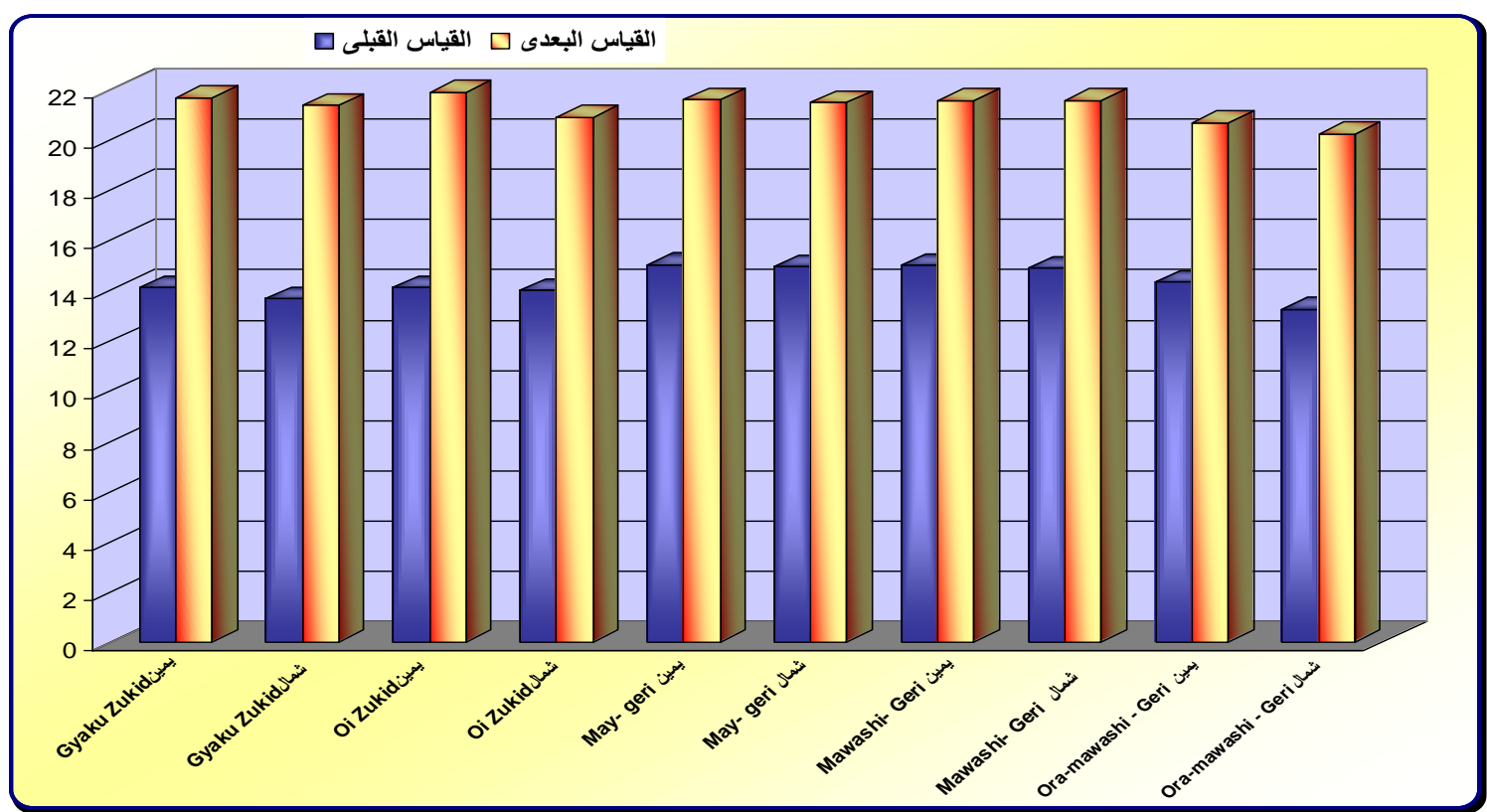

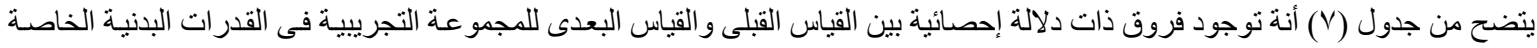

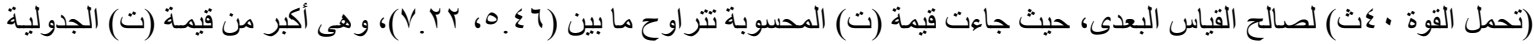

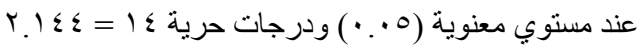




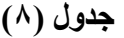

المتوسطات الحسابية والأنحراف المعيارى ودلالة الفروق (قيمة "ت") بين القياس القبلى والقياس البعدى للمجموعة التجريبية فى بعض $10=0$

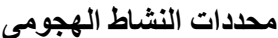

\begin{tabular}{|c|c|c|c|c|c|c|}
\hline \multirow{2}{*}{ قيمة "تلاتتها" } & \multicolumn{2}{|c|}{ القياس البعدى } & \multicolumn{2}{|c|}{ القياس القبلى } & \multirow{2}{*}{\multicolumn{2}{|c|}{ محددات النشاط الهجومى }} \\
\hline & $r \varepsilon$ & $r^{p}$ & $\varepsilon$ & م & & \\
\hline 1.14 & $r . r q$ & $V . \leqslant 0$ & 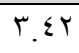 & $\vee . \wedge 1$ & مداخل الهجوم سو - نو - سن & \multirow{3}{*}{ مداخل } \\
\hline $1 . \wedge \wedge$ & 1.70 & T.I & r.IV & r.79 & مداخل الهجوم تاي- نو - سن & \\
\hline$* r$.VA &. .99 & .94 & 1.01 & 1.17 & مداخل الهجوم جو - نو - سن & \\
\hline$* Y .09$ & 1.0 & 0.11 & $1 . v 1$ & $r .01$ & فعالية النشاط الهجومى فى الدقيقة الأولى من المبار اة & \multirow{4}{*}{ النشالية } \\
\hline *Y.YI & $1.1 \leq$ & 0.14 & 1.20 & r. $\leqslant \wedge$ & فعالية النشاط الهجومى فى الدقيقة الثانية من المبار اة & \\
\hline$* \varepsilon . \wedge 1$ & 1.94 & 0.19 & $1, r_{1}$ & t.AT & فعالية النشاط الهجومى فى الدقيقة الثالثة من المبار اة & \\
\hline$* \wedge . \wedge \vee$ & r.Ar & 17,17 & $r \leqslant 1$ & $9 . \wedge 1$ & فعالية النشاط الهجو مى في المجموع الكلي للمبار اة & \\
\hline
\end{tabular}

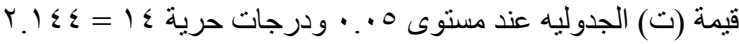

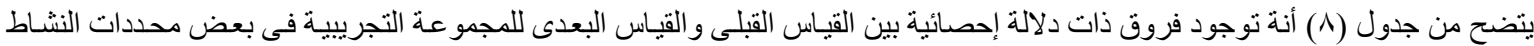

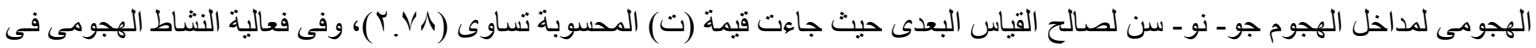

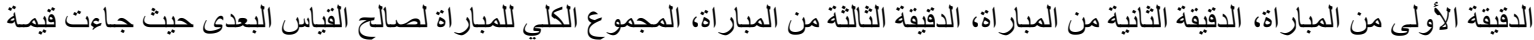

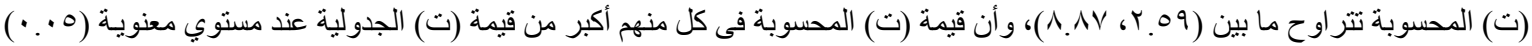

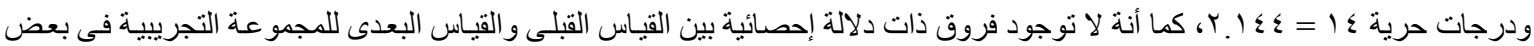

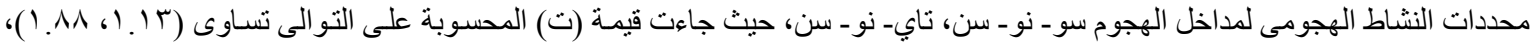

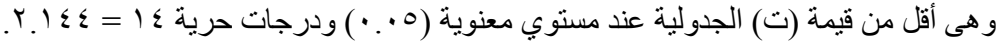

شكل (§) ( )

يوضح المتوسطات الحسابية بين القياس القبلى والبعدي للمجموعة التجريبية فى بعض محددات النشاط الهجومى

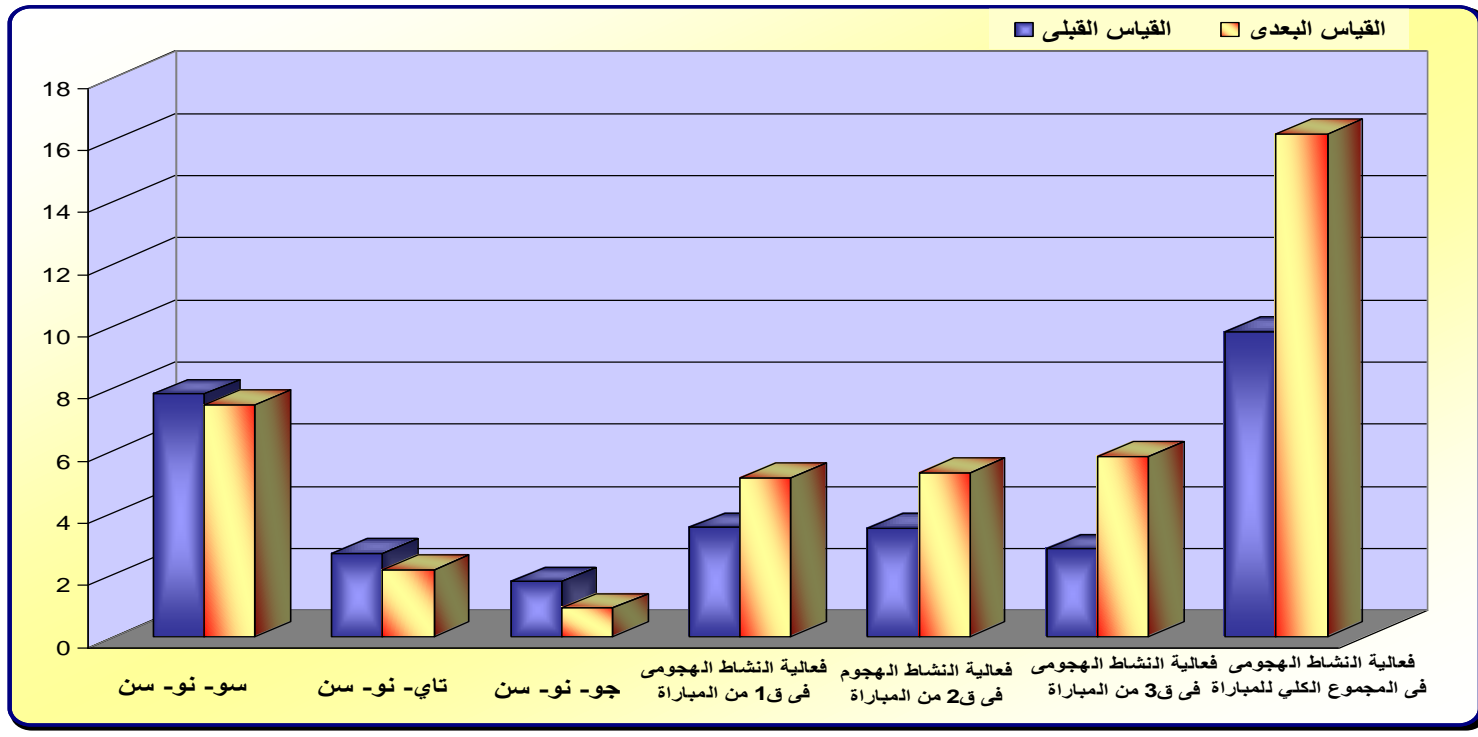

مناقثة النتائج :

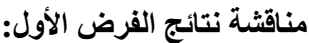

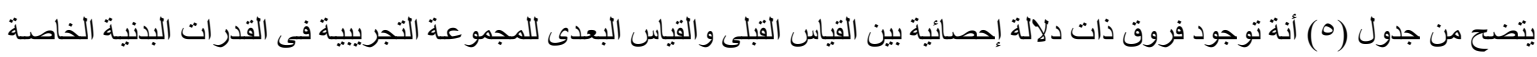

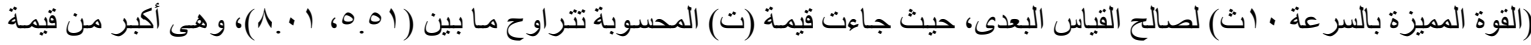

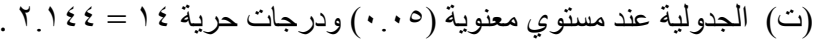




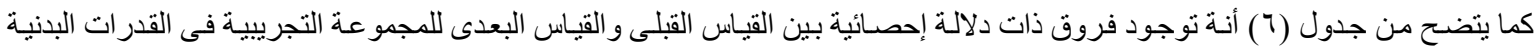

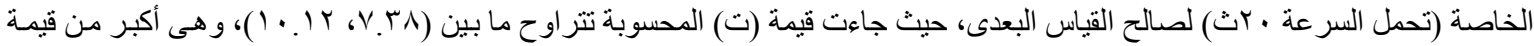

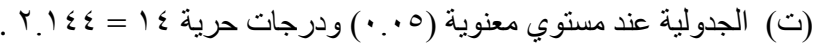

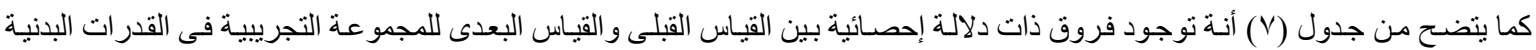

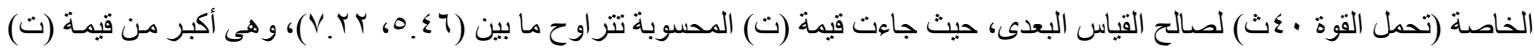

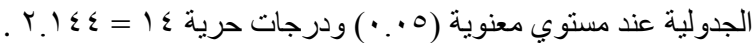

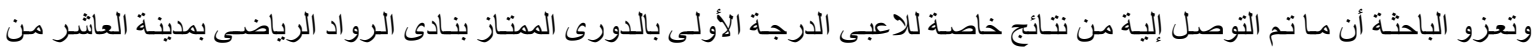

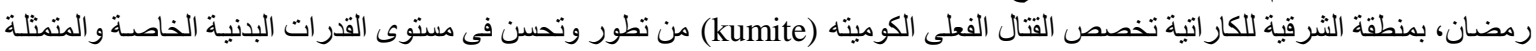

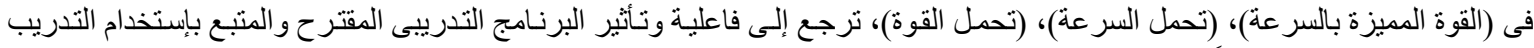

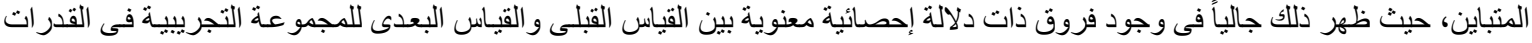

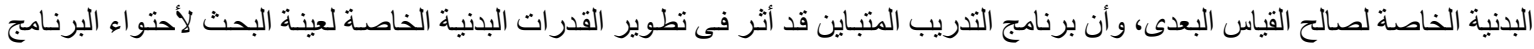

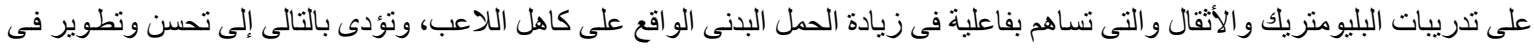

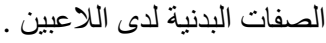

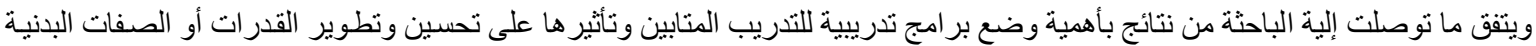

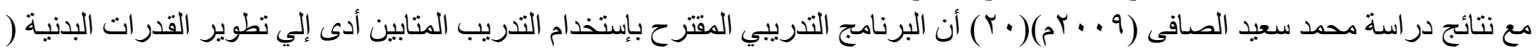

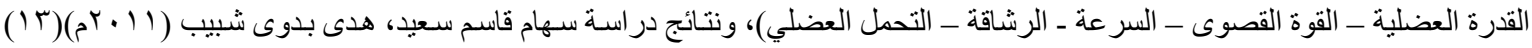

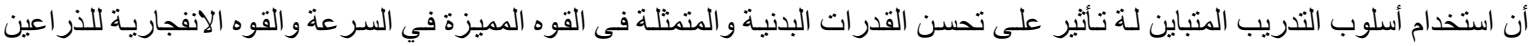

و والرجلين.

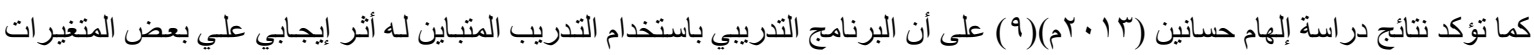
البدنية لدي لاعبات رمي الرمح.

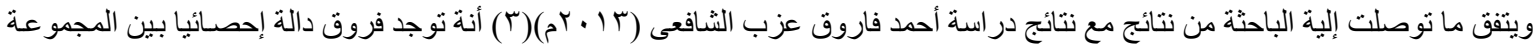

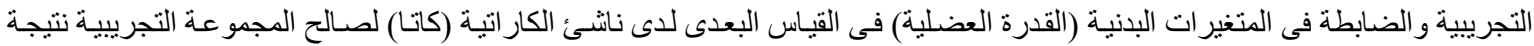

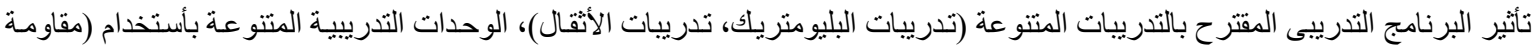

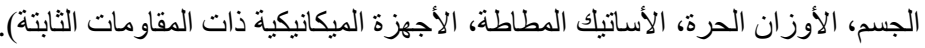

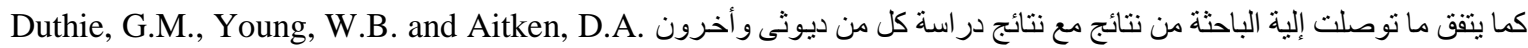

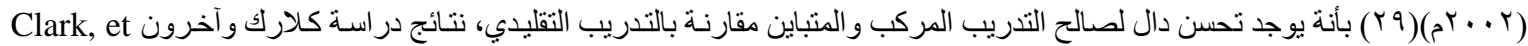

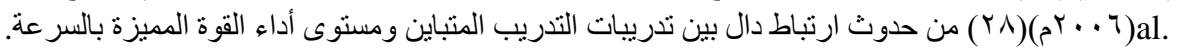

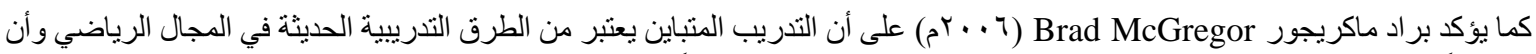

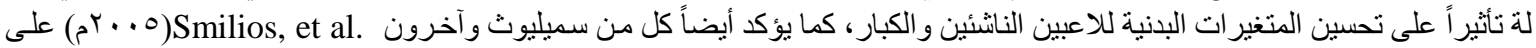

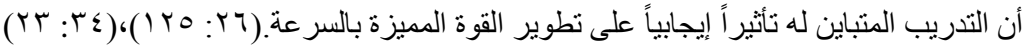

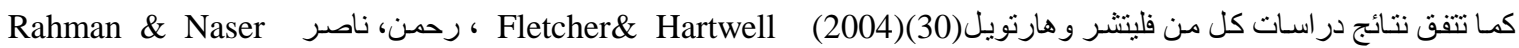

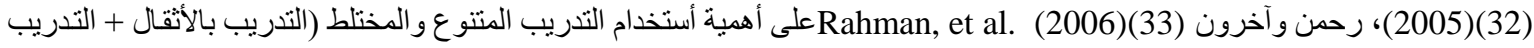

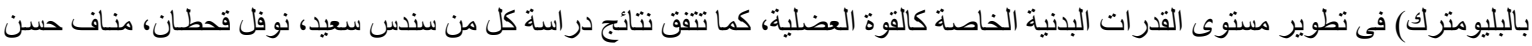

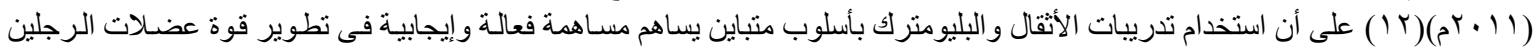

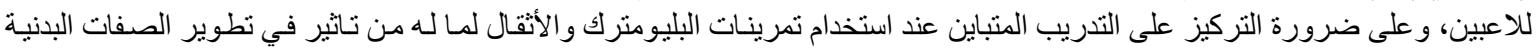

الخاصة.

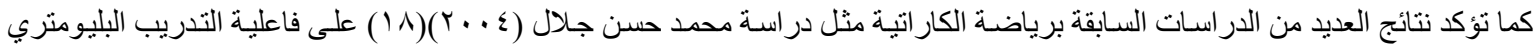

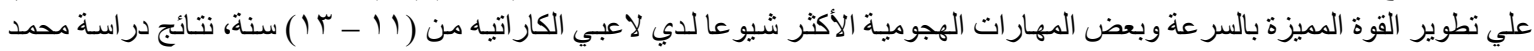

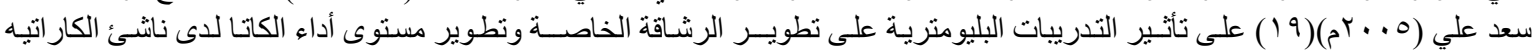

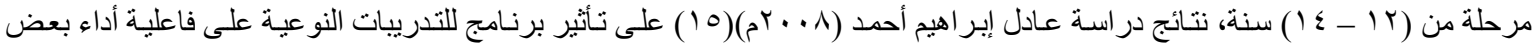

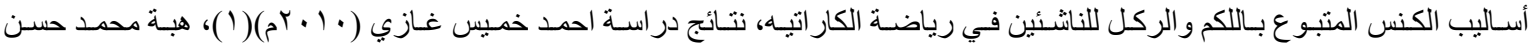

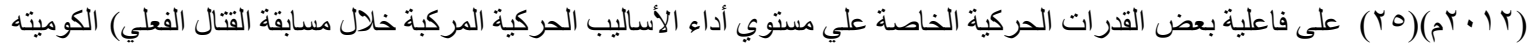

- kumite ). 


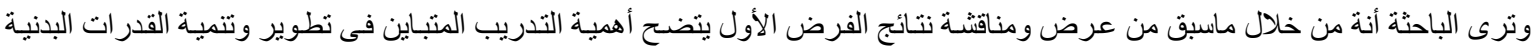

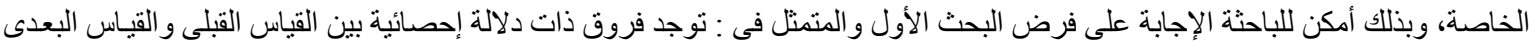

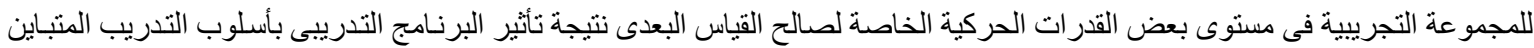

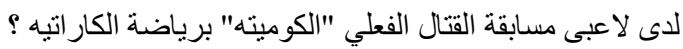

مناقشة نتائج الفرض الثانى:

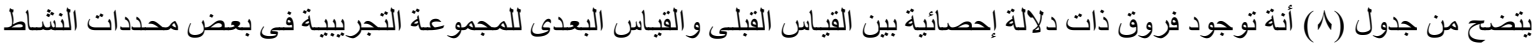

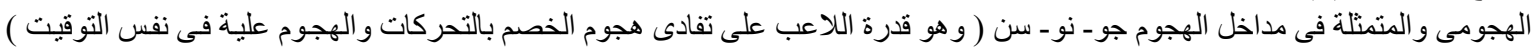

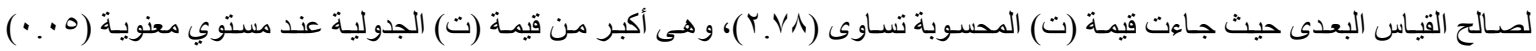

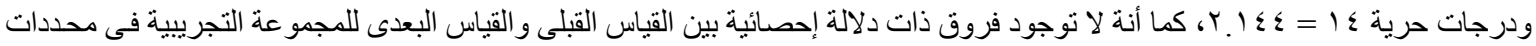

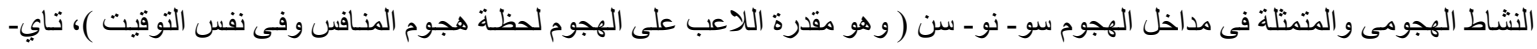

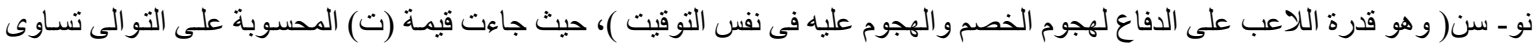

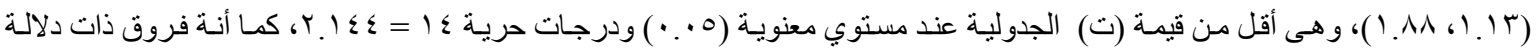

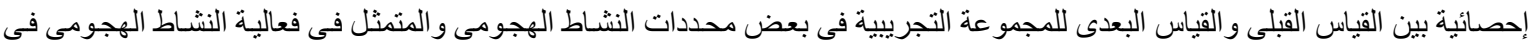

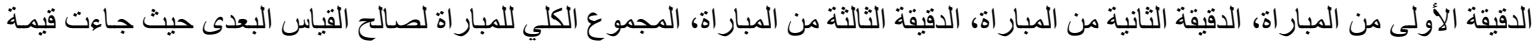

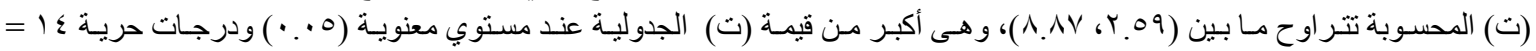

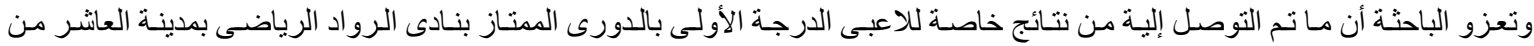

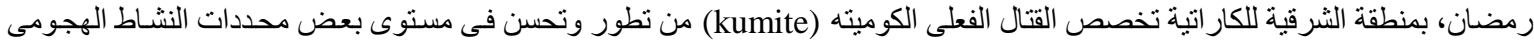

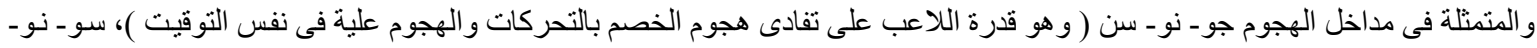

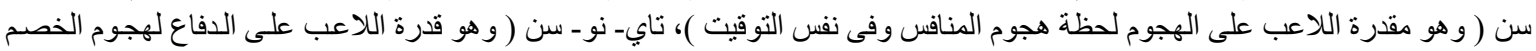

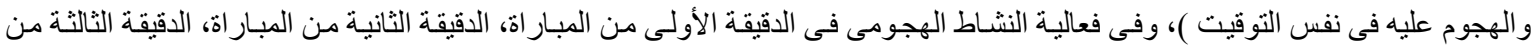

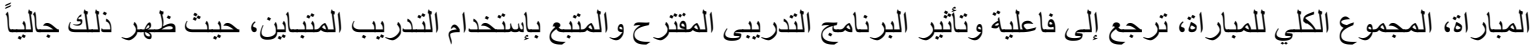

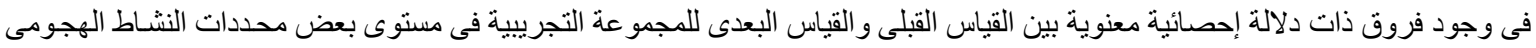

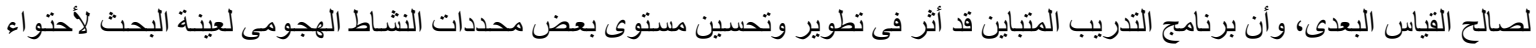

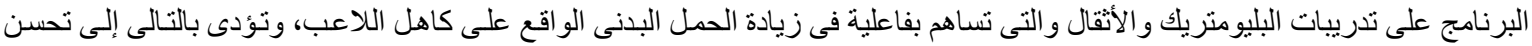

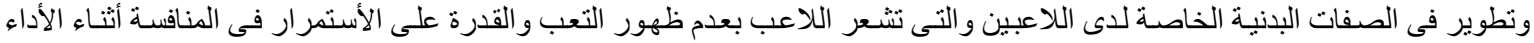

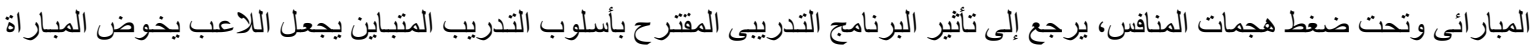

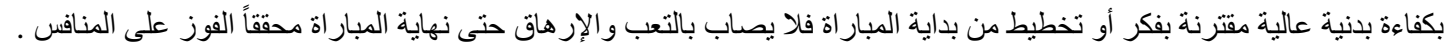

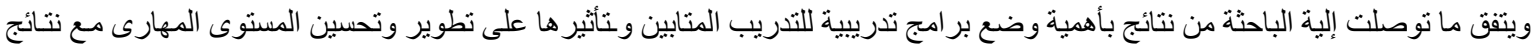

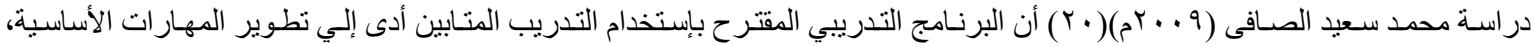

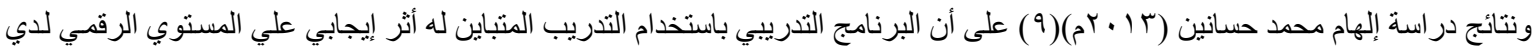
لاعبات رمي الرمح.

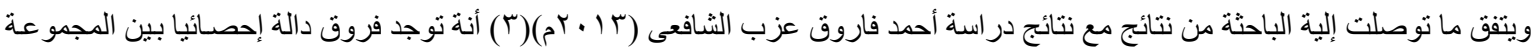

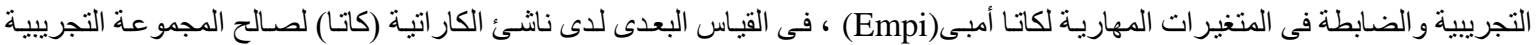

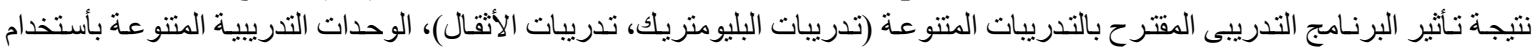

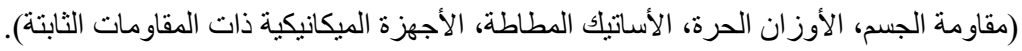

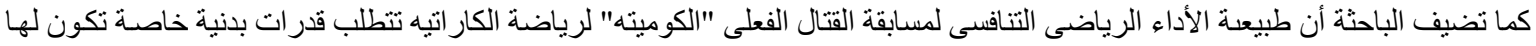

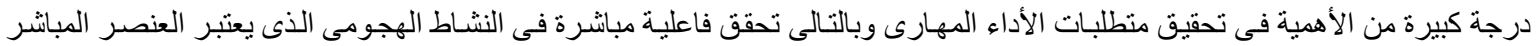

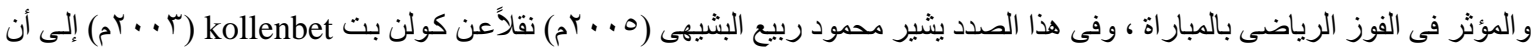

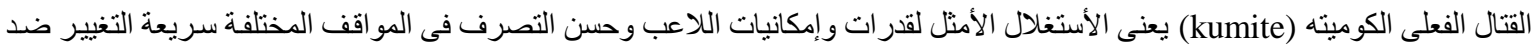

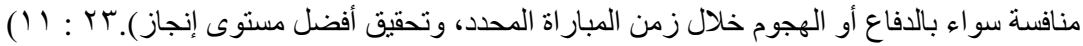

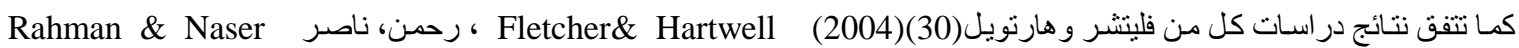

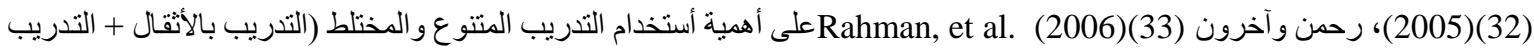
بالبليو منرك) فى تطوير مستوى الأداء المهارى، وحن. 


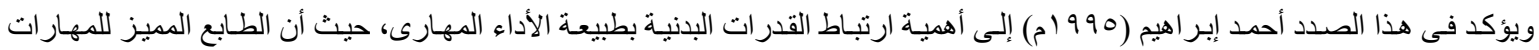

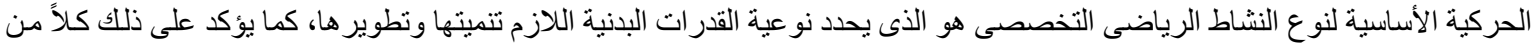

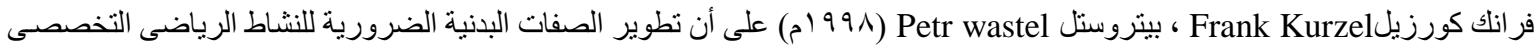

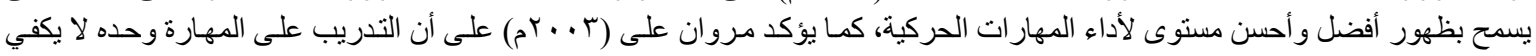

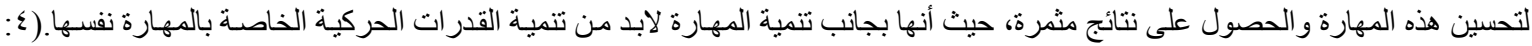

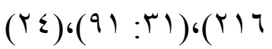

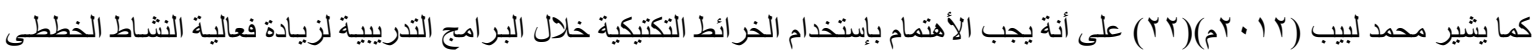
لكلاعبى رياضة الكار اتية.

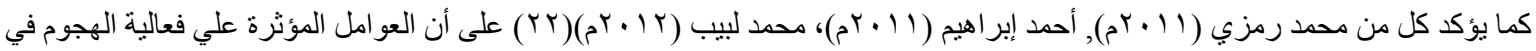

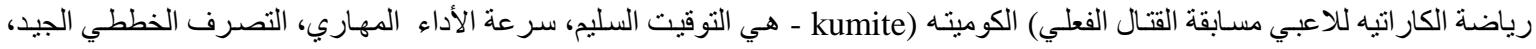

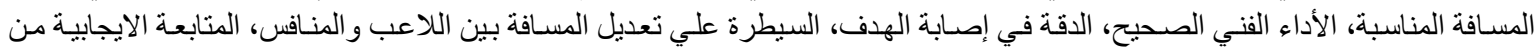

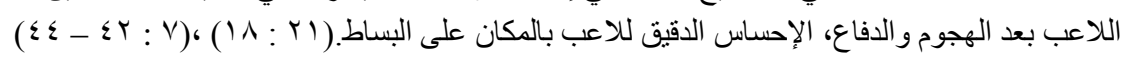

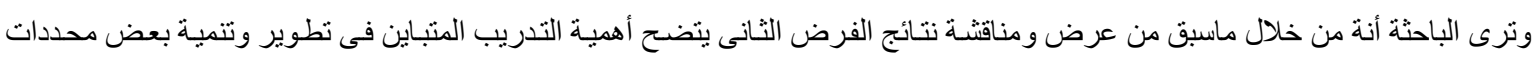

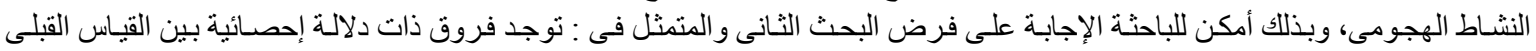

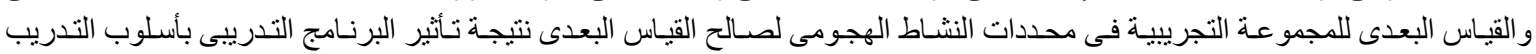
المتباين لاى لاعبى مسابقة القتال الفعلي "الكوميته" برياضة الكيدة الكار اتيه ؟

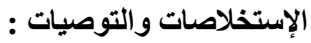

الإستخلاصات :

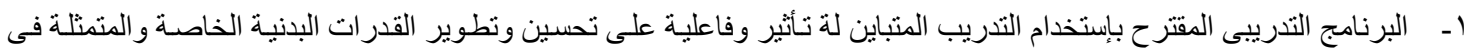

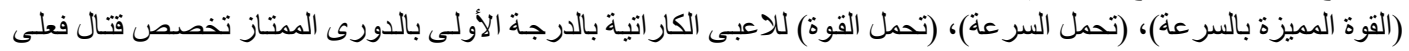

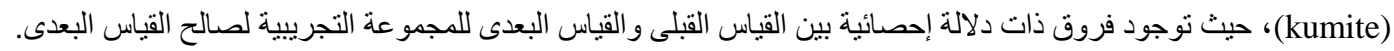

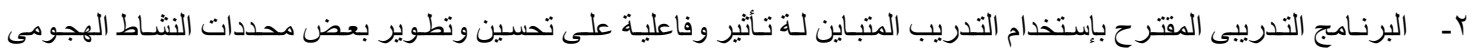

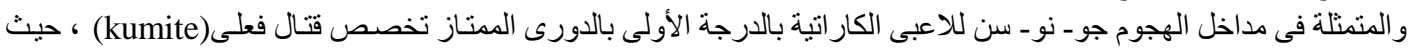

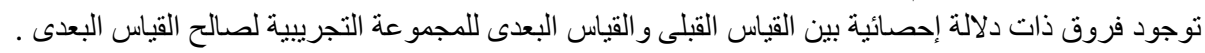

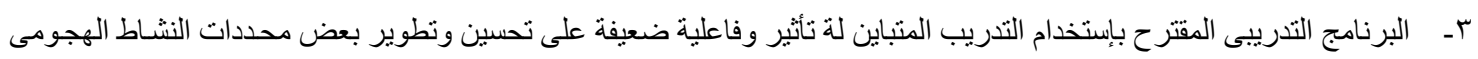

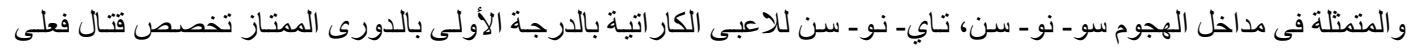

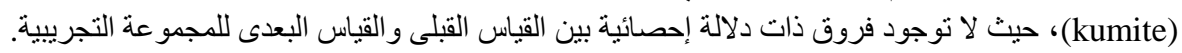

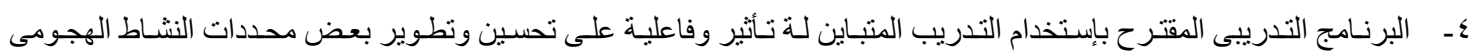

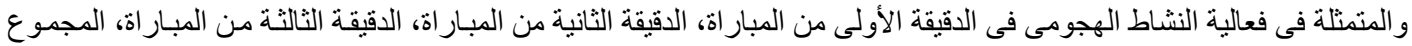

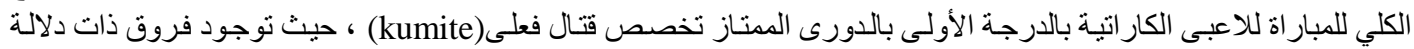

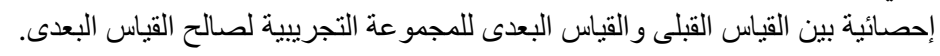

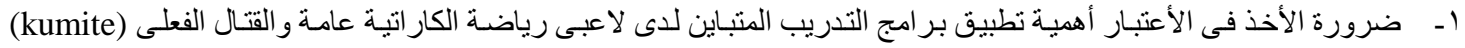

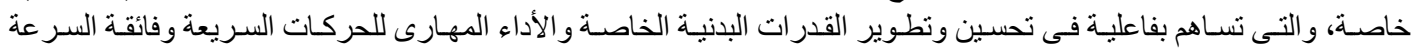

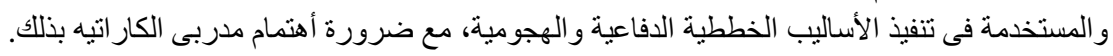

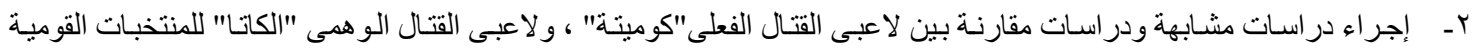

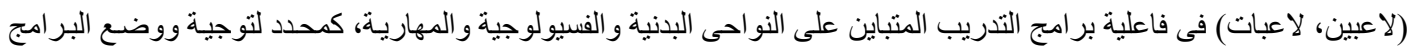




\section{أولاً :المراجع العربية:}

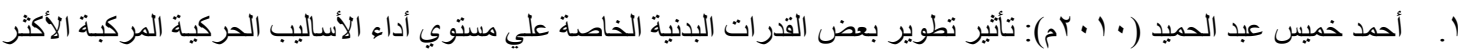

استخداما خلال مسابقة القتال الفعلي لرياضة الكار اتيه، رسالة ماجستير، كلية التربية الرياضية للبنين، جامعة الإسكندرية.

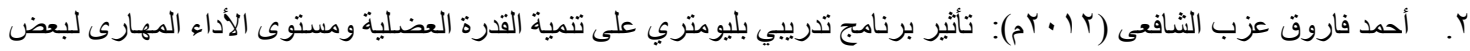

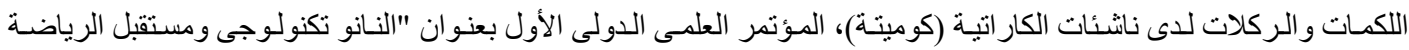

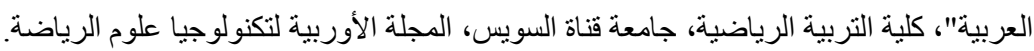

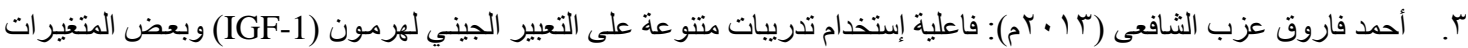

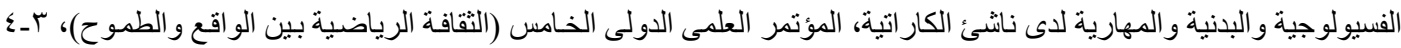
نيسان (إبريل)، كلية التربية الرياضية، جامعة اليرموك، الأردن.

ع. أحمد محمود إبر اهيم (990 (1)): مبادئ التخطيط للبر امج التعليمية و التدريبية لرياضة الكار اتيه، منشأة المعارف، الإسكندرية.

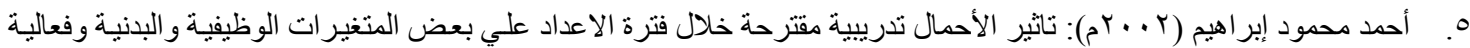

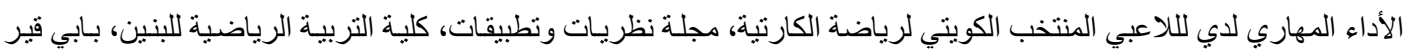

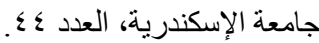
أحمد محمود إبر اهيم (0 . بrم): موسو عة محددات التدريب (النظريـة والتطبيق) لتخطبط البرامج التدريبية لرياضـة الكار اتيه، منشأة المعارف، الإسكندرية.

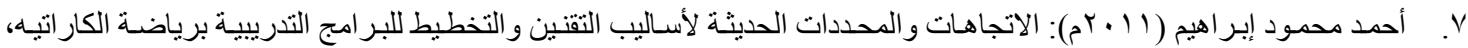
منشأة المعارف، الإسكندرية. أحمد محمود إبر اهيم (r ا ـ rم): الموسوعة العلمية و التطبيقية " الاتجاهات و المحددات الحديثـة لأساليب التقنين و التخطبط للبر امج التدرييية برياضة الكار اتيه "، منشاة المعارف، الإسكندرية.

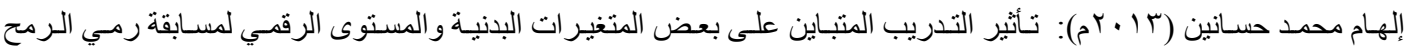
.9 لطالبات كلية التربية الرياضية بالمنيا، رسالة ماجستير، كلية التربية الرياضية، جامعة المنيا. • 1. حين علي العلي, عامر فاخر شغاتي (• ( • rم): استر اتيجيات طر ائق و أساليب التنريب الرياضي, مكتب النور, بغداد، العر اق.

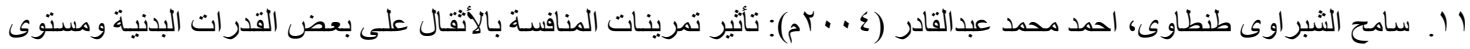
الأداء المهاري فى رياضة الكار اتيه، المجلة العلمية للبحوث و الدراسات، العدد الثامن، كلية التربية الرياضية ببورسعيد، جامعة قناة السويس.

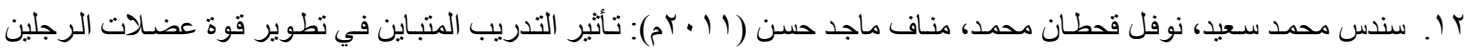

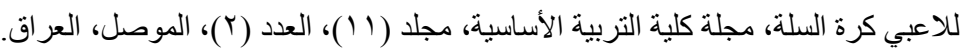

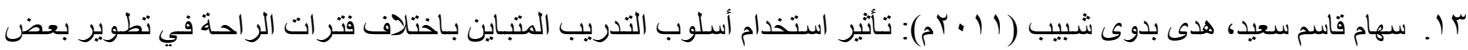
القدرات البدنية و الوظيفية بالكرة الطائرة، مجلة كلية التربية الرياضية، جامعة ديالى، بغداد، العراق. ع ا. شريف عبد القادر العوضي، عمر محمد لبيب (ع + - بم): قو اعد الهجوم، بدون. 10 في رياضة الكار اتيه، رسالة ماجستير، كلية التربية الرياضية للبنين، جامعة المنصورة. 
T ا. عصام عبد الحميد ( . . . rم): تأثير استخدام بعض الأساليب الفسيولوجية لتقنين حمل التدريب على كفاءة الجهاز الدوري التنفسي

وبعض المتغير ات البدنية والمهارية لدى ناشئ كرة القدم، رسالة دكتور اه، كلية التربية الرياضية، جامعة المنيا.

V V. على فهمي البيك، عماد الدين عباس، محمد أحمد عبده خليل (9 . . rم): طرق وأسـاليب التدريب لتنمية وتطوير القدرات اللاهو ائية

و الهوائية، منشأة المعارف، مصر.

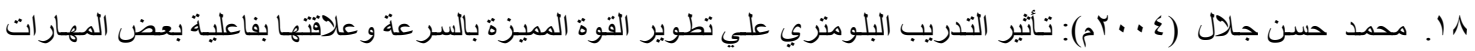

الهجوميـة الأكثر شـيو عاً لـدي لاعبـي الكار اتيـه مرحلـة (1 (1 1 ) سـنة، رسـالة ماجسـتير كليـة التربيـة الرياضـية بنـين، جامعـة

الإسكندرية.

9 ا . محمد سعد علي (0 . . Үم): تأتثير التدريبات البليومترية على تطويـر الرشـاقة الخاصــة وعلاقتها بتطوير مستوى أداء الكاتـا لدى

ناثئ الكار اتيه مرحلة من r ا ـ ـ ا سنة، رسالة دكتور اه غير منشورة، كلية التربية الرياضية للبنين جامعة الإسكندرية.

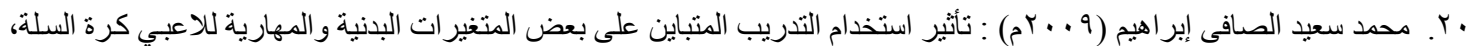

رسالة ماجستير، كلية التربية الرياضية، جامعة المنيا.

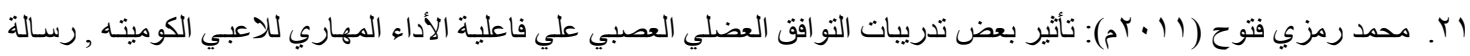

ماجستير غير منشورة , كلية التربية الرياضية , جامعة المنصورة.

r r. محمد لبيب عبد العزيز (r ا • rم): الخر ائط التكتيكيـة وفعاليـة إستخدام مداخل الهجوم لدى لاعبى مسـابقة القتال الفعلي "كوميته"

كمرشد لتخطيط البر امج برياضة الكار اتيه, رسالة ماجستير غير منشورة , كلية التربية الرياضية للبنين, جامعة بنها.

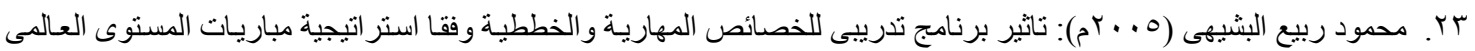

على مستوى اداء نتائج لاعبى رياضة الكار اتيه، رسالة دكتور اة كلية التربية الرياضية للبنين بالهرم ، جامعة حلوان.

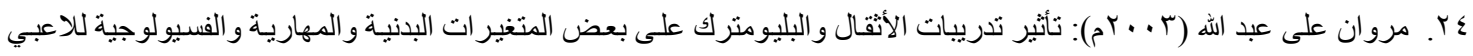

كرة اليد، رسالة دكتور اه، كلية التربية الرياضية، جامعة المنيا.

ه r. هبه محمد حسن (r ا • r م): تأثير التدريب المركب المتوازي لتطوير القدرات الحركية الخاصة علي مستوي استخدام بعض توقيتات

المداخل الهجو ميـة وفعاليـة السـلوك الهجومي للدي لاعبي رياضـة الكار اتيه, رسـالة ماجستير غير منشورة, كلية التربيـة الرياضية

للبنين, جامعة الإسكندرية

\section{ثُانياً :المراجع الأجنبية:}

26. Brad McGregor (2006): the application of complex training for the development of explosive power, Journal of Strength and Conditioning Research, 14(3), pp: 360.

27. Chip Sigmon (2003): 52-week Basketball Training, Human Kinetics

28. Clark RA, Bryant AL, Reaburn P. (2006):The acute effects of a single set of contrast preloading on a loaded countermovement jump training session, J Strength Cond Res. Feb;20(1):162-6.

29. Duthie, G.M., Young, W.B. and Aitken, D.A. (2002): The acute effects of heavy loads on jump squat performance: an evaluation of the complex and contrast methods of power development, Journal of Strength and Conditioning Research, 16 (4) pp: 530-538.

30. Fletcher, I.M., and M. Hartwell (2004): Effect of an 8-Week Combined Weights and Plyometrics Training Program on Golf Drive Performance, The Journal of Strength and Conditioning Research, Vol. 18, No. 1, pp. 59-62. 
31. Frank, K and peter, W. (1998): Fitness boxing, sterling Co., Inc. corporate.

32. Rahman Rahimi, Naser Behpur (2005): The effects of plyometric, weight and plyometric - weight training on anaerobic power and muscular strength, Physical Education and Sport Vol. 3, No 1, pp. $81-91$

33. Rahman Rahimi, Parvin Arshadi, Naser Behpur, Saeed Sadeghi Boroujerdi, Mohammad Rahimi (2006): evaluation of plyometrics, weight training and their combination on angular velocity , Physical Education and Sport, Vol. 4, No 1, pp. 1 - 8

34. Smilios Ilias, Theophilos Pilianidis, Konstantinos Sotiropoulos, Manolis Antonakis, Savvas P Tokmakidis (2005): Short-term effects of selected exercise and load in contrast training on vertical jump performance, J Strength Cond Res. 2005 Feb ;19 (1): Greece

35. www.Iraq sport academy 


\section{الملخص باللغة العربيـة}

فاعلية التدريب المتباين على مستوى بعض القدر ات الحركية الخاصة ومحددات النشـاط الهجومى لدى لاعبى مسـابقة القتال الفعلي "الكوميته" برياضئة الكار اتيه. نجلاء أمين رزق الطناحى الريات قسم الرياضات المائية والمنازلات ـ كلية التربية الرياضية للبنات ـ جامعة الزقازيق - جمهورية مصر العربية.

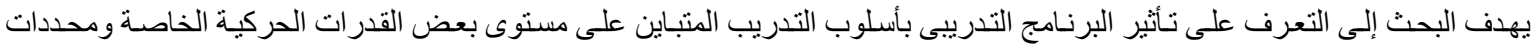

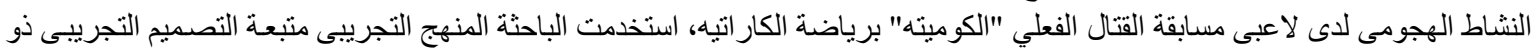

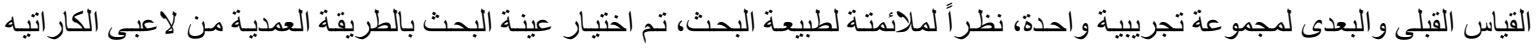

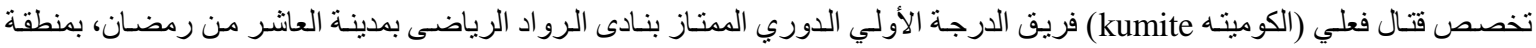

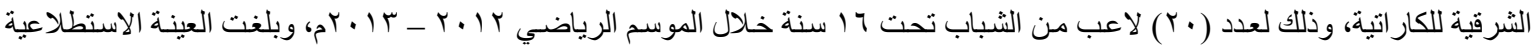

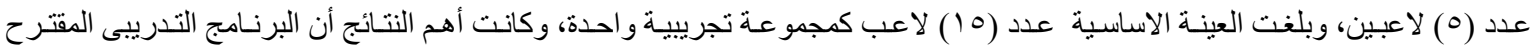

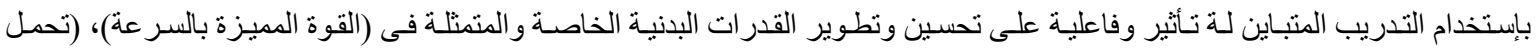

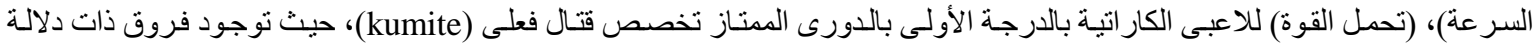

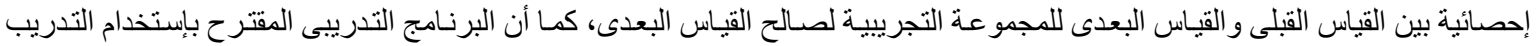

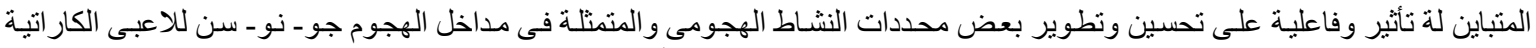

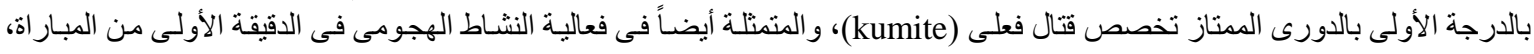

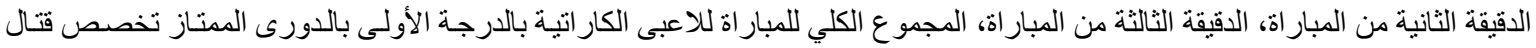

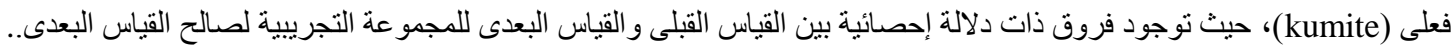


Effectiveness of Contrast training on the level of some special movement abilities and determinants of attacking activity for Players of Contest The Aetual Fighting " Kumite" on the sport of karate.

\section{Nagla EI Tanahi}

The research aims to identify the impact of the Contrast training on the level of some special movement abilities and determinants of attacking activity for Players of Contest The Actual Fighting " Kumite" on the sport of karate, The researcher used the experimental method followed a tribal measurement experimental design and post for a trial period of one, because of the relevance of the nature of the research, was selected sample way intentional players karate specialty actual fighting (kumite) first class team Premier League Club pioneers sports in the Tenth of Ramadan, area East Karate, and for a number (20) player of the youth under 16 years old during the sports season from 2012 to $2013 \mathrm{~m}$, and the total sample exploratory number (5) players, and reached the basic sample number (15) for the player as a group experimental one, and The most important results that the proposed Training Program using disparate training has an impact and effectiveness of the improvement and development of physical capabilities represented in (distinctive strengths speed), (bearing speed), (bearing force) of karate players primarily Premier League allocate actual combat (kumite), where There is statistically significant differences between the measurement pre and post test measurement differences experimental group for post-measurement, and The proposed program is the training using the differential training has an impact and effectiveness of the improvement and development of some of the determinants of offensive activity represented at the entrances to the attack Jo no-enactment of karate players primarily Premier League allocate actual combat (kumite), represented also in the effectiveness of offensive activity in the first minute of the game, the second minute of the game, the third minute of the game, the total match of karate players primarily Premier League allocate actual combat (kumite), where There is statistically significant differences between the measurement of tribal and telemetric experimental group in favor of telemetric. 
